\title{
The unsteady flow of a weakly compressible fluid in a thin porous layer. I: Two-dimensional theory
}

Article

Published Version

Needham, D. J., Langdon, S., Busswell, G. S. and Gilchrist, J. P. (2009) The unsteady flow of a weakly compressible fluid in a thin porous layer. I: Two-dimensional theory. SIAM Journal on Applied Mathematics (SIAP), 69 (4). pp. 1084-1109. ISSN 0036-1399 doi: https://doi.org/10.1137/070703405 Available at https://centaur.reading.ac.uk/4648/

It is advisable to refer to the publisher's version if you intend to cite from the work. See Guidance on citing.

To link to this article DOI: http://dx.doi.org/10.1137/070703405

Publisher: Society for Industrial and Applied Mathematics

All outputs in CentAUR are protected by Intellectual Property Rights law, including copyright law. Copyright and IPR is retained by the creators or other copyright holders. Terms and conditions for use of this material are defined in the End User Agreement.

www.reading.ac.uk/centaur 
Central Archive at the University of Reading

Reading's research outputs online 


\title{
THE UNSTEADY FLOW OF A WEAKLY COMPRESSIBLE FLUID IN A THIN POROUS LAYER I: TWO-DIMENSIONAL THEORY*
}

\author{
D. J. NEEDHAM ${ }^{\dagger}$, S. LANGDON LA $^{\ddagger}$ G. S. BUSSWELL ${ }^{\S}$, AND J. P. GILCHRIST ${ }^{\S}$
}

\begin{abstract}
We consider the problem of determining the pressure and velocity fields for a weakly compressible fluid flowing in a two-dimensional reservoir in an inhomogeneous, anisotropic porous medium, with vertical side walls and variable upper and lower boundaries, in the presence of vertical wells injecting or extracting fluid. Numerical solution of this problem may be expensive, particularly in the case that the depth scale of the layer $h$ is small compared to the horizontal length scale $l$. This is a situation which occurs frequently in the application to oil reservoir recovery. Under the assumption that $\epsilon=h / l \ll 1$, we show that the pressure field varies only in the horizontal direction away from the wells (the outer region). We construct two-term asymptotic expansions in $\epsilon$ in both the inner (near the wells) and outer regions and use the asymptotic matching principle to derive analytical expressions for all significant process quantities. This approach, via the method of matched asymptotic expansions, takes advantage of the small aspect ratio of the reservoir, $\epsilon$, at precisely the stage where full numerical computations become stiff, and also reveals the detailed structure of the dynamics of the flow, both in the neighborhood of wells and away from wells.
\end{abstract}

Key words. oil recovery, thin porous layer, matched asymptotics

AMS subject classifications. 35K15, 35K20, 76M45, 76S05, 86A99

DOI. $10.1137 / 070703405$

1. Introduction. It is standard practice in the oil and gas industry to use reservoir simulators based on numerical methods such as the finite difference or finite element techniques. This kind of approach has been shown to be enormously successful over the years in modeling a wide variety of physical processes in the reservoir e.g., faults, rock layering effects, complex fluid phase behavior, etc. While reservoir simulators of this type will continue to play a crucial role in the industry, it is well known that to use them takes considerable expertise and time. Because of the numerical nature of the modeling process, gridding, time-stepping, and convergence issues require care and attention. Long execution times are often necessary for certain types of problems, e.g., hydraulically fractured wells.

Analytic techniques, for the reasons outlined, can therefore play a valuable role in the industry. Such techniques, although they may have some simplifying assumptions, allow a reservoir or production engineer to perform a quick study of their reservoir in order to obtain a broad understanding of the dynamical processes and make approximate costing forecasts. Analytic solutions are extremely fast and provide none of the timestepping and convergence issues seen with a numerically based simulator. Also, a necessary step in many reservoir studies involves the history matching of observed data by optimizing model parameters. The history matched model is then used for

\footnotetext{
* Received by the editors September 21, 2007; accepted for publication (in revised form) October 6, 2008; published electronically January 28, 2009.

http://www.siam.org/journals/siap/69-4/70340.html

${ }^{\dagger}$ School of Mathematics, University of Birmingham, Birmingham B15 2TT, UK (d.j.needham@ bham.ac.uk).

${ }^{\ddagger}$ Department of Mathematics, University of Reading, Reading RG6 6AX, UK (s.langdon@reading. ac.uk).

§Schlumberger Oilfield UK Plc, Wyndyke Furlong, Abingdon, OX14 1UJ, UK (gbusswell@ hotmail.co.uk, pgilchrist@abingdon.oilfield.slb.com).
} 
performance prediction. Given the speed and reliability of analytic results, there is a clear opportunity to exploit their use in history matching studies.

There has been much work in the literature regarding analytic approaches, particularly for well testing applications $[10,5,15,1,9]$ but also from a full field reservoir standpoint, where multiple wells and reservoir boundaries must be accounted for to forecast production over the required timescales. Algorithms for full field simulation problems based on analytic approaches have been presented in the literature for porous media with homogeneous and anisotropic permeability in a variety of sources $[4,19,13]$. A more complex problem involves the application of analytic approaches to full field scenarios where the reservoir has inhomogeneous permeability and variable geometry $[12,14,8,18]$.

In this paper we introduce a new approach to solving full field reservoir problems with inhomogeneous and anisotropic permeability and variable reservoir geometry using the method of matched asymptotic expansions. Specifically, our problem involves determining analytical expressions for the pressure and velocity fields for a weakly compressible fluid flowing in a horizontal reservoir with variable upper and lower boundaries. Vertical wells injecting or extracting fluid from the reservoir can be considered as line sources and sinks, respectively. Numerical solution of the full equations of motion throughout the reservoir can be prohibitively expensive. However, under the condition that the depth scale of the reservoir $h$ is small compared to the length scale of the reservoir $l$, as is often the case in geophysical applications, it can be shown (allowing further that the porous medium has inhomogeneous and anisotropic permeability) that the dimension of the problem can be reduced away from the wells, with solution of the full equations of motion being required only in a small domain around the wells where the geometry is radically simplified. Moreover, as the ratio $h / l$ decreases, efficient application of numerical schemes becomes harder, while the problem becomes more amenable to solution via matched asymptotic theory.

Here, we restrict attention to the case of two-dimensional flow. The full threedimensional problem will be dealt with in subsequent work [11]. We introduce the parameter $\epsilon=h / l$ and consider asymptotic solutions to the equations of motion of the fluid in increasing powers of $\epsilon$, with $0<\epsilon \ll 1$. In the vicinity of a well (the inner region) the pressure field is two-dimensional, but away from the wells (the outer region) the pressure field is only one-dimensional. This immediately leads to a reduction in complexity. Here, however, rather than solving the full equations of motion numerically in the inner and outer regions, we construct two-term expansions in both the inner and outer regions. These expansions in the inner and outer regions can then be matched, via the Van Dyke asymptotic matching principle [20], enabling us to derive amenable analytical expressions for all significant process quantities.

We begin in section 2 by deriving the equations of motion in the porous medium. Conservation of mass and momentum lead to a strongly parabolic linear initial boundary value problem for the dynamic fluid pressure (from which the fluid velocity field can be deduced), with Neumann boundary conditions, under the assumption that the walls are impenetrable to the fluid in the porous medium. This initial boundary value problem has a unique solution, but its direct computation would be expensive, primarily due to stiffness when $0<\epsilon \ll 1$. We thus consider the associated steady state problem [SSP], a linear strongly elliptic Neumann problem, which also has a unique solution (up to a constant) under the further constraint that the sum of the total volume fluxes at the wells (the line sources and sinks) is zero. Solution of the steady state problem is considered in section 3. Subtracting the solution of the steady state problem from the solution of the initial value problem leads to a strongly parabolic 
homogeneous problem with no discontinuities across the sources and sinks. The solution of this problem leads to a regular self-adjoint eigenvalue problem [EVP] whose solution is considered in section 4.

Rather than solving [SSP] and [EVP] directly, the solution to each problem is considered in the asymptotic limit $\epsilon \rightarrow 0$, via the method of matched asymptotic expansions. For the two-dimensional problem these asymptotic solutions can be constructed analytically. To solve [SSP], we proceed first with the situation when the wells are well spaced and are away from the reservoir boundaries, after which the case of wells close to a boundary, or close together, is considered in sections 3.1 and 3.2. The asymptotic solution can be constructed directly in the outer region, up to $O\left(\epsilon^{2}\right)$. In the inner region, determination of the leading order terms reduces to the solution of a strongly elliptic problem whose solution can be written analytically in terms of the eigenvalues and corresponding eigenfunctions of a regular Sturm-Liouville eigenvalue problem. The asymptotic solution of [EVP] in section 4 also reduces to a regular Sturm-Liouville eigenvalue problem identical in structure to that discussed in section 3 , and a consideration of this allows us to demonstrate that the solution to the full initial boundary value problem approaches the solution to the steady state problem through terms exponentially small with respect to time $t$ as $t \rightarrow \infty$. With $D_{z}$ being the permeability scale in the vertical direction and $D_{x}$ being the permeability scale in the horizontal direction, the further generalization that $D_{z}=o\left(D_{x}\right)$ rather than $O\left(D_{x}\right)$ is considered in section 5 , where it is shown that the structure of the solution is identical to that found for the case that $D_{z}=O\left(D_{x}\right)$, after a suitable redefinition of the parameter $\epsilon$. The constraint on the sum of the total volume fluxes at the wells being zero is removed in section 6 , and in section 7 we apply the theory to a simple model example. Finally in section 8 we draw some conclusions.

2. Equations of motion. We consider the flow of a weakly compressible fluid in the presence of sources and sinks in a reservoir of porous medium with variable upper and lower boundary. The reservoir has permeability which is inhomogeneous and anisotropic. We restrict attention to the situation when the flow is two-dimensional. We denote the interior of the porous medium by $M \subset \mathbb{R}^{2}$ and its impermeable boundary by $\partial M \subset \mathbb{R}^{2}$, with $\bar{M}=M \cup \partial M$. We introduce rectangular Cartesian coordinates $(x, z)$, with $z$ pointing vertically upwards and $x$ pointing horizontally. The vertical side walls of the reservoir are taken to be at $x= \pm l$, with $l>0$. The upper and lower surface of the reservoir are described by $z=h z_{+}(x / l)$ and $z=h z_{-}(x / l)$, respectively, for $x \in[-l, l]$, with $h(>0)$ being the reservoir depth scale and $z_{+}, z_{-}:[-1,1] \mapsto \mathbb{R}$ being such that $z_{+}, z_{-} \in C^{1}([-1,1])$ and $z_{+}(x)>z_{-}(x)$ for all $x \in[-1,1]$. Normal fields on the upper and lower surfaces are then given by $\mathbf{n}_{+}(x)=\left(-\frac{h}{l} z_{+}^{\prime}(x), 1\right)$ and $\mathbf{n}_{-}(x)=\left(\frac{h}{l} z_{-}^{\prime}(x),-1\right)$, respectively, for $x \in[-1,1]$, with the normals directed out of $\bar{M}$. The situation is illustrated in Figure 2.1.

Embedded within $\bar{M}$ are $N(\in \mathbb{N})$ vertical line sources/sinks at locations $x_{i} \in$ $(-l, l), i=1, \ldots, N$. Each line source/sink extends from the upper surface to the lower surface of $\bar{M}$ and represents a model of a vertical bore hole in the reservoir extending from the upper to the lower surface of the reservoir and extracting or injecting fluid along its whole length. This model is standard in the oil industry $[3,10]$. The prescribed strength of each line source/sink then represents the details of the controlled volumetric extraction mechanism in the bore hole along its length. The two components of permeability, in the $x$ - and $z$-directions, respectively, are given by

$$
D_{0} D_{x}\left(\frac{x}{l}, \frac{z}{h}\right) \geq D_{m}>0, \quad D_{0} D_{z}\left(\frac{x}{l}, \frac{z}{h}\right) \geq D_{m}>0, \quad(x, z) \in \bar{M},
$$




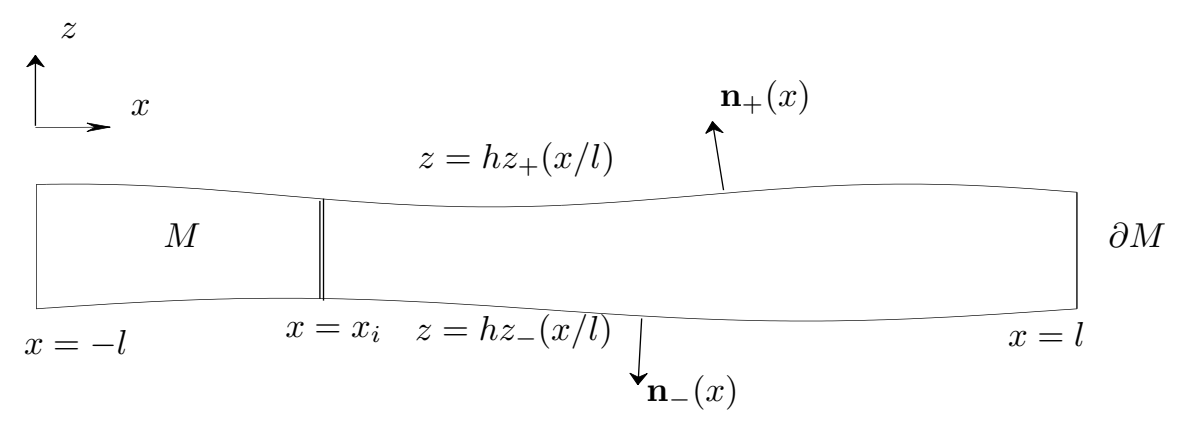

FIG. 2.1. Porous layer $M \subset \mathbb{R}^{2}$, with impermeable boundary $\partial M$.

with $D_{x}, D_{z}: \bar{M} \mapsto \mathbb{R}^{+}$such that

$$
D_{x}, D_{z} \in C^{1}(\bar{M}) .
$$

Here $D_{0}>0$ is a permeability scale for the layer and $D_{m}>0$ is a lower bound on permeability in the layer. To avoid confusion, we emphasise here that subscripts $x$ and $z$ attached to the functions $D_{x}$ and $D_{z}$ do not indicate partial differentiation but merely denote the permeability direction.

We represent the fluid velocity field and pressure field at each point within the layer by $\mathbf{q}=\mathbf{q}(\mathbf{r}, t)=(u(\mathbf{r}, t), w(\mathbf{r}, t))$ and $p=p(\mathbf{r}, t)$, respectively, for each $(\mathbf{r}, t) \in$ $\bar{M} \times[0, \infty)$. Here $t \geq 0$ represents time. The equation of conservation of fluid mass within the layer is then

$$
\rho_{t}+(\rho u)_{x}+(\rho w)_{z}=\sum_{i=1}^{N} \rho s_{i}\left(\frac{z}{h}\right) \frac{1}{l} \delta\left(\frac{x-x_{i}}{l}\right), \quad(x, z) \in M, \quad t \in(0, \infty) .
$$

Here $\delta: \mathbb{R} \mapsto \mathbb{R}$ is the usual Dirac delta function, $\rho=\rho(\mathbf{r}, t)$ is the fluid density field for $(\mathbf{r}, t) \in \bar{M} \times[0, \infty)$, and the line source/sink volumetric strengths are represented by $s_{i}:\left[z_{-}\left(\frac{x_{i}}{l}\right), z_{+}\left(\frac{x_{i}}{l}\right)\right] \mapsto \mathbb{R}, i=1, \ldots, N$. From practical considerations of the employed volumetric extraction mechanisms in bore holes, it is reasonable to take

$$
s_{i} \in C\left(\left[z_{-}\left(\frac{x_{i}}{l}\right), z_{+}\left(\frac{x_{i}}{l}\right)\right]\right), \quad i=1, \ldots, N .
$$

The total volume flux per unit width from the $i$ th line source/sink is then

$$
Q_{i}=\int_{h z_{-}\left(x_{i} / l\right)}^{h z_{+}\left(x_{i} / l\right)} s_{i}\left(\frac{\lambda}{h}\right) \mathrm{d} \lambda, \quad i=1, \ldots, N .
$$

Conservation of momentum in the fluid is accounted for through the D'Arcy equation for flow in a porous media, giving

$$
u=-D_{0} D_{x}\left(\frac{x}{l}, \frac{z}{h}\right) p_{x}, \quad w=-D_{0} D_{z}\left(\frac{x}{l}, \frac{z}{h}\right)\left(p_{z}+\rho g\right)
$$

for all $(x, z) \in M, t \in(0, \infty)$, where $g$ is the acceleration due to gravity. The effect of weak compressibility is accounted for through the equation of state,

$$
\rho(p)=\rho_{0}\left(1+c_{t}\left(p-p_{0}\right)\right)
$$


with $c_{t}>0$ being a constant isothermal expansion coefficient and $\rho_{0}$ and $p_{0}$ being positive reference density and pressure, respectively. Now, for a weakly compressible fluid, $0<c_{t} p_{0} \ll 1$, and the weakly compressible model is obtained by replacing $\rho(p)$ by its dominant contribution from (2.7) in both of the equations of motion (2.3) and (2.6). We obtain as our final model for the flow in the reservoir

$$
\begin{gathered}
c_{t} p_{t}+u_{x}+w_{z}=\sum_{i=1}^{N} s_{i}\left(\frac{z}{h}\right) \frac{1}{l} \delta\left(\frac{x-x_{i}}{l}\right), \\
u=-D_{0} D_{x}\left(\frac{x}{l}, \frac{z}{h}\right) p_{x}, \\
w=-D_{0} D_{z}\left(\frac{x}{l}, \frac{z}{h}\right)\left(p_{z}+\rho_{0} g\right)
\end{gathered}
$$

for all $(x, z) \in M, t \in(0, \infty)$. The equations of motion (2.8)-(2.10) form the basis for established models for weakly compressible flows in porous reservoirs [2], and we will take (2.8)-(2.10) as the model for the flow in the porous reservoir throughout the rest of the paper. We now set

$$
Q=\sum_{i=1}^{N}\left|Q_{i}\right| \quad(>0) .
$$

The natural scales are then $x \sim l$ and $z \sim h$, from the geometry of the porous layer, while $s_{i} \sim Q / h$, via (2.5). The continuity equation (2.8) then requires $u \sim Q / h$ and $w \sim Q / l$, while the momentum equation $(2.9)$ requires $p \sim(l Q) /\left(h D_{0}\right)$. We therefore introduce the dimensionless variables,

$$
x=l x^{\prime}, z=h z^{\prime}, s_{i}=\frac{Q}{h} s_{i}^{\prime}, u=\frac{Q}{h} u^{\prime}, w=\frac{Q}{l} w^{\prime}, p=\left(\frac{l Q}{h D_{0}}\right) p^{\prime}, t=\frac{c_{t} l^{2}}{D_{0}} t^{\prime} .
$$

On substitution from (2.12) into (2.8)-(2.10) (and dropping primes for convenience) we obtain the dimensionless equations of motion as

$$
\begin{gathered}
\bar{p}_{t}+u_{x}+w_{z}=\sum_{i=1}^{N} s_{i}(z) \delta\left(x-x_{i}\right), \\
u=-D_{x}(x, z) \bar{p}_{x}, \\
\epsilon^{2} w=-D_{z}(x, z) \bar{p}_{z}
\end{gathered}
$$

for all $(x, z) \in M^{\prime}, t \in(0, \infty)$. Here, $p(x, z, t)=-\hat{\sigma} z+\bar{p}(x, z, t)$, with $\bar{p}$ being the dynamic fluid pressure, and the dimensionless parameters $\epsilon$ and $\hat{\sigma}$ are given by $\epsilon=h / l$ and $\hat{\sigma}=h^{2} \rho_{0} g D_{0} /(l Q)$. The values of the model parameters will vary depending upon the details of the reservoir under consideration. However, for a typical field scenario the values $h \sim 200 \mathrm{~m}, l \sim 20,000 \mathrm{~m}, Q \sim 2 \mathrm{~m}^{3} / \mathrm{s}, c_{t} \sim 1.45 \times 10^{-9} \mathrm{~m}^{2} / \mathrm{N}$, $p_{0} \sim 2.76 \times 10^{7} \mathrm{~N} / \mathrm{m}^{2}$, and $D_{0} \sim 10^{-10} \mathrm{~m}^{4} / \mathrm{Ns}$ may be considered as representative. This gives a value for $c_{t} p_{0} \sim 0.04$, which is entirely consistent with the adoption of the weakly compressible model proposed earlier. More significantly, the aspect ratio of a typical reservoir gives $\epsilon \sim 0.01$ (and this may be as small as $10^{-3}$ for large reservoirs). The dimensionless domain is now

$$
M^{\prime}=\left\{(x, z) \in \mathbb{R}^{2}: x \in(-1,1), z \in\left(z_{-}(x), z_{+}(x)\right)\right\},
$$


with closure $\bar{M}^{\prime}$ and boundary $\partial M^{\prime}$. The line source/sink locations are at $x_{i} \in(-1,1)$, $i=1, \ldots, N$. The volume flux condition (2.5) becomes

$$
\alpha_{i}=\int_{z_{-}\left(x_{i}\right)}^{z_{+}\left(x_{i}\right)} s_{i}(\mu) \mathrm{d} \mu, \quad i=1, \ldots, N
$$

with $\alpha_{i}=Q_{i} / Q$, and hence $\left|\alpha_{i}\right|=\left|Q_{i}\right| / Q \leq 1, i=1, \ldots, N$, and $\sum_{i=1}^{N}\left|\alpha_{i}\right|=$ $\sum_{i=1}^{N}\left|Q_{i}\right| / Q=1$, via (2.11). We next consider the boundary conditions. On the boundary $\partial M^{\prime}$ the walls are impenetrable to the fluid in the porous layer. Thus

$$
\begin{gathered}
u(-1, z, t)=0 \text { for all } z \in\left[z_{-}(-1), z_{+}(-1)\right], t \in(0, \infty), \\
\quad u(1, z, t)=0 \text { for all } z \in\left[z_{-}(1), z_{+}(1)\right], t \in(0, \infty), \\
w\left(x, z_{+}(x), t\right)-z_{+}^{\prime}(x) u\left(x, z_{+}(x), t\right)=0 \text { for all } x \in(-1,1), t \in(0, \infty), \\
w\left(x, z_{-}(x), t\right)-z_{-}^{\prime}(x) u\left(x, z_{-}(x), t\right)=0 \quad \text { for all } x \in(-1,1), t \in(0, \infty) .
\end{gathered}
$$

Finally we have the initial condition

$$
\bar{p}(x, z, 0)=\bar{p}_{0}(x, z) \quad \text { for all }(x, z) \in \bar{M}^{\prime},
$$

with $\bar{p}_{0} \in C\left(\bar{M}^{\prime}\right) \cap P C^{1}\left(\bar{M}^{\prime}\right)$, where $P C^{1}\left(\bar{M}^{\prime}\right)$ represents the class of piecewise continuously differentiable functions on $\bar{M}^{\prime}$. The full problem for consideration is given by (2.13)-(2.15), (2.16)-(2.20), which we will refer to as [IBVP]. To proceed it is convenient to introduce $d_{i}=\left\{(x, z) \in \bar{M}^{\prime}: x=x_{i}, z \in\left(z_{-}\left(x_{i}\right), z_{+}\left(x_{i}\right)\right)\right\} \subset \bar{M}^{\prime}$, for each $i=1, \ldots, N$, and $d=\bigcup_{i=1}^{N} d_{i}$. We require that a solution to [IBVP] has the following regularity, which is classical in the framework of the Dirac delta function formalism:

(i) $\bar{p} \in C\left(\bar{M}^{\prime} \times[0, \infty)\right) \cap C^{1}\left(\left(\bar{M}^{\prime} \backslash \bar{d}\right) \times(0, \infty)\right) \cap C^{2}\left(\left(M^{\prime} \backslash d\right) \times(0, \infty)\right)$, $u \in C\left(\left(\bar{M}^{\prime} \backslash \bar{d}\right) \times(0, \infty)\right) \cap C^{1}\left(\left(M^{\prime} \backslash d\right) \times(0, \infty)\right)$, $w \in C\left(\bar{M}^{\prime} \times(0, \infty)\right) \cap C^{1}\left(\left(M^{\prime} \backslash d\right) \times(0, \infty)\right)$;

(ii) $\lim _{x \rightarrow x_{i}^{ \pm}} \bar{p}_{x}$ and $\lim _{x \rightarrow x_{i}^{ \pm}} \bar{p}_{z}$ both exist uniformly for $z \in\left[z_{-}\left(x_{i}\right), z_{+}\left(x_{i}\right)\right]$ at each $t \in(0, \infty), i=1, \ldots, N$

(iii) $\left[\bar{p}_{z}\right]_{x_{i}^{-}}^{x^{+}}=0,\left[\bar{p}_{x}\right]_{x_{i}^{-}}^{x_{+}^{+}}=-s_{i}(z) / D_{x}\left(x_{i}, z\right)$ for all $z \in\left[z_{-}\left(x_{i}\right), z_{+}\left(x_{i}\right)\right]$ at each $t \in(0, \infty), i=1, \ldots, N$.

We observe that (ii) requires, via (2.14), (2.15), that $\lim _{x \rightarrow x_{i}^{ \pm}} u$ and $\lim _{x \rightarrow x_{i}^{ \pm}} w$ both exist uniformly for $z \in\left[z_{-}\left(x_{i}\right), z_{+}\left(x_{i}\right)\right]$, and (iii) requires that

$$
[u]_{x_{i}^{-}}^{x_{+}^{+}}=s_{i}(z), \quad[w]_{x_{i}^{-}}^{x_{+}^{+}}=0
$$

for all $z \in\left[z_{-}\left(x_{i}\right), z_{+}\left(x_{i}\right)\right]$ at each $t \in(0, \infty), i=1, \ldots, N$. We now have the following preliminary result concerning [IBVP].

THEOREM 2.1. For each $\epsilon>0$, [IBVP] has a unique solution $u, w, \bar{p}: \bar{M}^{\prime} \times$ $[0, \infty) \mapsto \mathbb{R}$. Moreover,

$$
\left(\iint_{\bar{M}^{\prime}} \bar{p}(x, z, t) \mathrm{d} x \mathrm{~d} z\right)_{t}=\sum_{i=1}^{N} \alpha_{i}
$$

for all $t \in(0, \infty)$. 
Proof. Existence and uniqueness follow via regularity (2.2) and (2.4), with (2.1), on noting from (2.13)-(2.15) that [IBVP] is equivalent to the scalar initial boundary value problem

$$
\begin{gathered}
\bar{p}_{t}-\left\{\left(D_{x}(x, z) \bar{p}_{x}\right)_{x}+\left(\epsilon^{-2} D_{z}(x, z) \bar{p}_{z}\right)_{z}\right\}=\sum_{i=1}^{N} s_{i}(z) \delta\left(x-x_{i}\right), \\
\qquad(x, z) \in M^{\prime}, \quad t \in(0, \infty), \\
\bar{p}_{x}(-1, z, t)=0 \text { for all } z \in\left[z_{-}(-1), z_{+}(-1)\right], t \in(0, \infty), \\
\bar{p}_{x}(1, z, t)=0 \text { for all } z \in\left[z_{-}(1), z_{+}(1)\right], t \in(0, \infty), \\
D_{z}\left(x, z_{+}(x)\right) \bar{p}_{z}\left(x, z_{+}(x), t\right)-\epsilon^{2} z_{+}^{\prime}(x) D_{x}\left(x, z_{+}(x)\right) \bar{p}_{x}\left(x, z_{+}(x), t\right)=0 \\
\quad \text { for all } x \in(-1,1), t \in(0, \infty), \\
D_{z}\left(x, z_{-}(x)\right) \bar{p}_{z}\left(x, z_{-}(x), t\right)-\epsilon^{2} z_{-}^{\prime}(x) D_{x}\left(x, z_{-}(x)\right) \bar{p}_{x}\left(x, z_{-}(x), t\right)=0 \\
\text { for all } x \in(-1,1), t \in(0, \infty), \\
\bar{p}(x, z, 0)=\bar{p}_{0}(x, z) \text { for all }(x, z) \in \bar{M}^{\prime} .
\end{gathered}
$$

The partial differential equation (2.22) is linear and strongly parabolic, with boundary conditions of nondegenerate (via (2.1)) weighted Neumann type, after which existence and uniqueness follow from the classical theory in [7, Chapter 3]. Equation (2.21) follows from an application of Green's theorem on the plane to (2.22) in $\bar{M}^{\prime}$ on using the associated boundary conditions and regularity in (i)-(iii).

The steady state problem associated with [IBVP] is

$$
\begin{gathered}
\hat{u}_{x}+\hat{w}_{z}=\sum_{i=1}^{N} s_{i}(z) \delta\left(x-x_{i}\right), \quad(x, z) \in M^{\prime}, \\
\hat{u}=-D_{x}(x, z) \hat{p}_{x}, \quad(x, z) \in M^{\prime}, \\
\epsilon^{2} \hat{w}=-D_{z}(x, z) \hat{p}_{z}, \quad(x, z) \in M^{\prime}, \\
\hat{u}(-1, z)=0 \quad \text { for all } z \in\left[z_{-}(-1), z_{+}(-1)\right], \\
\hat{u}(1, z)=0 \quad \text { for all } z \in\left[z_{-}(1), z_{+}(1)\right], \\
\hat{w}\left(x, z_{+}(x)\right)-z_{+}^{\prime}(x) \hat{u}\left(x, z_{+}(x)\right)=0 \quad \text { for all } x \in(-1,1), \\
\hat{w}\left(x, z_{-}(x)\right)-z_{-}^{\prime}(x) \hat{u}\left(x, z_{-}(x)\right)=0 \quad \text { for all } x \in(-1,1),
\end{gathered}
$$

which we will refer to as [SSP]. Corresponding to (i)-(iii), a solution to [SSP] has the following regularity:

(si) $\hat{p} \in C\left(\bar{M}^{\prime}\right) \cap C^{1}\left(\bar{M}^{\prime} \backslash \bar{d}\right) \cap C^{2}\left(M^{\prime} \backslash d\right), \hat{u} \in C\left(\bar{M}^{\prime} \backslash \bar{d}\right) \cap C^{1}\left(M^{\prime} \backslash d\right)$, $\hat{w} \in C\left(\bar{M}^{\prime}\right) \cap C^{1}\left(M^{\prime} \backslash d\right)$;

(sii) $\lim _{x \rightarrow x_{i}^{ \pm}} \hat{p}_{x}$ and $\lim _{x \rightarrow x_{i}^{ \pm}} \hat{p}_{z}$ both exist uniformly for $z \in\left[z_{-}\left(x_{i}\right), z_{+}\left(x_{i}\right)\right]$ for each $i \stackrel{=}{=} 1, \ldots, N$;

(siii) $\left[\hat{p}_{z}\right]_{x_{i}^{-}}^{x_{i}^{+}}=0,\left[\hat{p}_{x}\right]_{x_{i}^{-}}^{x_{i}^{+}}=-s_{i}(z) / D_{x}\left(x_{i}, z\right)$ for all $z \in\left[z_{-}\left(x_{i}\right), z_{+}\left(x_{i}\right)\right]$ and each $i=1, \ldots, N$.

As before, $\lim _{x \rightarrow x_{i}^{ \pm}} \hat{u}$ and $\lim _{x \rightarrow x_{i}^{ \pm}} \hat{w}$ exist uniformly for $z \in\left[z_{-}\left(x_{i}\right), z_{+}\left(x_{i}\right)\right]$, and

$$
[\hat{u}]_{x_{i}^{-}}^{x_{+}^{+}}=s_{i}(z), \quad[\hat{w}]_{x_{i}^{-}}^{x_{+}^{+}}=0
$$

for all $z \in\left[z_{-}\left(x_{i}\right), z_{+}\left(x_{i}\right)\right]$ and for each $i=1, \ldots, N$. Again, following standard theory for linear strongly elliptic weighted Neumann problems (see, for example, [7] or [17, Chapters 8, 9]), we have the following result. 
TheOREm 2.2. For each $\epsilon>0$, [SSP] has a unique (up to addition of a constant in $\hat{p}$ ) solution $\hat{u}, \hat{w}, \hat{p}: \bar{M}^{\prime} \mapsto \mathbb{R}$ if and only if

$$
\sum_{i=1}^{N} \alpha_{i}=0 .
$$

In what follows we will assume that the specified flux constants $\alpha_{i}, i=1, \ldots, N$, satisfy condition (2.31). Now let $\tilde{u}, \tilde{w}, \tilde{p}: \bar{M}^{\prime} \times[0, \infty) \mapsto \mathbb{R}$ be defined by $\tilde{u}=u-\hat{u}$, $\tilde{w}=w-\hat{w}, \tilde{p}=\bar{p}-\hat{p}$. It then follows that $\tilde{u}, \tilde{w}$, and $\tilde{p}$ are solutions to the problem

$$
\begin{gathered}
\tilde{p}_{t}-\left\{\left(D_{x}(x, z) \tilde{p}_{x}\right)_{x}+\left(\epsilon^{-2} D_{z}(x, z) \tilde{p}_{z}\right)_{z}\right\}=0,(x, z) \in M^{\prime}, t \in(0, \infty), \\
\tilde{p}_{x}(-1, z, t)=0 \text { for all } z \in\left[z_{-}(-1), z_{+}(-1)\right], t \in(0, \infty) \\
\tilde{p}_{x}(1, z, t)=0 \text { for all } z \in\left[z_{-}(1), z_{+}(1)\right], t \in(0, \infty) \\
D_{z}\left(x, z_{+}(x)\right) \tilde{p}_{z}\left(x, z_{+}(x), t\right)-\epsilon^{2} z_{+}^{\prime}(x) D_{x}\left(x, z_{+}(x)\right) \tilde{p}_{x}\left(x, z_{+}(x), t\right)=0 \\
\quad \text { for all } x \in(-1,1), t \in(0, \infty), \\
D_{z}\left(x, z_{-}(x)\right) \tilde{p}_{z}\left(x, z_{-}(x), t\right)-\epsilon^{2} z_{-}^{\prime}(x) D_{x}\left(x, z_{-}(x)\right) \tilde{p}_{x}\left(x, z_{-}(x), t\right)=0 \\
\quad \text { for all } x \in(-1,1), t \in(0, \infty), \\
\tilde{p}(x, z, 0)=\bar{p}_{0}(x, z)-\hat{p}(x, z)=\tilde{p}_{0}(x, z) \quad \text { for all }(x, z) \in \bar{M}^{\prime}
\end{gathered}
$$

with regularity

$$
\tilde{p} \in C\left(\bar{M}^{\prime} \times[0, \infty)\right) \cap C^{1}\left(\bar{M}^{\prime} \times(0, \infty)\right) \cap C^{2}\left(M^{\prime} \times(0, \infty)\right),
$$

after which

$$
\tilde{u}=-D_{x}(x, z) \tilde{p}_{x}, \quad \tilde{w}=-\epsilon^{-2} D_{z}(x, z) \tilde{p}_{z}, \quad(x, z) \in \bar{M}^{\prime} \times(0, \infty) .
$$

To fix the indeterminate constant in Theorem 2.2 , we will take $\hat{p}: \bar{M}^{\prime} \mapsto \mathbb{R}$ to be that steady state which satisfies

$$
\iint_{\bar{M}^{\prime}} \hat{p}(x, z) \mathrm{d} x \mathrm{~d} z=\iint_{\bar{M}^{\prime}} \bar{p}_{0}(x, z) \mathrm{d} x \mathrm{~d} z=: I_{0}
$$

so that, via (2.37),

$$
\iint_{\bar{M}^{\prime}} \tilde{p}_{0}(x, z) \mathrm{d} x \mathrm{~d} z=0
$$

Now it follows from Theorem 2.1 that the strongly parabolic problem (2.32)-(2.39) has a unique solution in $\bar{M}^{\prime} \times[0, \infty)$. We will now construct this solution. To this end we first consider the following self-adjoint eigenvalue problem in $\bar{M}^{\prime}$ :

$$
\begin{gathered}
\left(D_{x}(x, z) \phi_{x}\right)_{x}+\left(\epsilon^{-2} D_{z}(x, z) \phi_{z}\right)_{z}+\lambda \phi=0, \quad(x, z) \in M^{\prime}, \\
\phi_{x}(-1, z)=0 \text { for all } z \in\left[z_{-}(-1), z_{+}(-1)\right], \\
\phi_{x}(1, z)=0 \text { for all } z \in\left[z_{-}(1), z_{+}(1)\right], \\
D_{z}\left(x, z_{+}(x)\right) \phi_{z}\left(x, z_{+}(x)\right)-\epsilon^{2} z_{+}^{\prime}(x) D_{x}\left(x, z_{+}(x)\right) \phi_{x}\left(x, z_{+}(x)\right)=0 \text { for all } x \in(-1,1), \\
D_{z}\left(x, z_{-}(x)\right) \phi_{z}\left(x, z_{-}(x)\right)-\epsilon^{2} z_{-}^{\prime}(x) D_{x}\left(x, z_{-}(x)\right) \phi_{x}\left(x, z_{-}(x)\right)=0 \text { for all } x \in(-1,1) .
\end{gathered}
$$

We will denote this eigenvalue problem by [EVP], with $\lambda \in \mathbb{C}$ being the eigenvalue parameter. It follows from (2.1) that this is a regular, self-adjoint eigenvalue problem. 
It then follows from standard theory that the eigenvalues of [EVP] are all real and given by $\lambda=\lambda_{j}(\epsilon), j=0,1,2, \ldots$, with

$$
0=\lambda_{0}(\epsilon)<\lambda_{1}(\epsilon)<\lambda_{2}(\epsilon)<\cdots
$$

and $\lambda_{j}(\epsilon) \rightarrow+\infty$ as $j \rightarrow \infty$. Each corresponding eigenspace is spanned by a single eigenfunction $\phi_{j}: \bar{M}^{\prime} \mapsto \mathbb{R}, j=0,1,2, \ldots$, with

$$
\phi_{0}(x, z, \epsilon)=\left(\operatorname{meas}\left(\bar{M}^{\prime}\right)\right)^{-1 / 2} \text { for all }(x, z) \in \bar{M}^{\prime},
$$

and meas $\left(\bar{M}^{\prime}\right)$ being the measure (area) of $\bar{M}^{\prime} \subset \mathbb{R}^{2}$. Moreover,

$$
\left\langle\phi_{i}, \phi_{j}\right\rangle=\iint_{\bar{M}^{\prime}} \phi_{i}(x, z, \epsilon) \phi_{j}(x, z, \epsilon) \mathrm{d} x \mathrm{~d} z=\delta_{i j},
$$

for $i, j=0,1,2, \ldots$, and $\delta_{i j}$ being the Kronecker delta symbol. Moreover, any function $\psi: \bar{M}^{\prime} \mapsto \mathbb{R}$ such that $\psi \in C\left(\bar{M}^{\prime}\right) \cap P C^{1}\left(\bar{M}^{\prime}\right)$ and satisfies the same boundary conditions on $\partial M^{\prime}$ as the set of eigenfunctions has the representation

$$
\psi(x, z)=\sum_{r=0}^{\infty} \psi_{r}(\epsilon) \phi_{r}(x, z, \epsilon), \quad(x, z) \in \bar{M}^{\prime},
$$

with the convergence of the sum being uniform and absolute for $(x, z) \in \bar{M}^{\prime}$, where

$$
\psi_{j}(\epsilon)=\left\langle\psi, \phi_{j}\right\rangle=\iint_{\bar{M}^{\prime}} \psi(x, z) \phi_{j}(x, z, \epsilon) \mathrm{d} x \mathrm{~d} z,
$$

for $j=0,1,2, \ldots$ (see, for example, [17, Chapters 8,9$]$ ). It is now straightforward to establish that the (unique) solution to (2.32)-(2.37) is given by

$$
\tilde{p}(x, z, t)=\sum_{n=1}^{\infty} a_{n}(\epsilon) \mathrm{e}^{-\lambda_{n}(\epsilon) t} \phi_{n}(x, z, \epsilon), \quad(x, z) \in \bar{M}^{\prime}, t \in[0, \infty),
$$

with $a_{0}(\epsilon)=0$, via $(2.37),(2.41),(2.43),(2.44)$, and $(2.45)$, and

$$
a_{n}(\epsilon)=\iint_{\bar{M}^{\prime}} \tilde{p}_{0}(u, v) \phi_{n}(u, v, \epsilon) \mathrm{d} u \mathrm{~d} v
$$

for $n=1,2, \ldots$ We observe immediately from (2.46), with (2.42), that $\tilde{p}(x, z, t) \rightarrow 0$ as $t \rightarrow \infty$, uniformly for all $(x, z) \in \bar{M}^{\prime}$, and also that $\tilde{p}_{x}(x, z, t), \tilde{p}_{z}(x, z, t) \rightarrow 0$ as $t \rightarrow \infty$, uniformly for all $(x, z) \in \bar{M}^{\prime}$. Thus, we have established the following result.

Theorem 2.3. Let $\alpha_{i}, i=1, \ldots, N$, be such that $\sum_{i=1}^{N} \alpha_{i}=0$. Then for each $\epsilon>0,[\mathrm{IBVP}]$ has a unique solution $u, w, \bar{p}: \bar{M}^{\prime} \times[0, \infty) \mapsto \mathbb{R}$ given by

$$
\begin{aligned}
& \bar{p}(x, z, t)=\hat{p}(x, z)+\tilde{p}(x, z, t), \\
& u(x, z, t)=\hat{u}(x, z)-D_{x}(x, z) \tilde{p}_{x}(x, z, t), \\
& w(x, z, t)=\hat{w}(x, z)-\epsilon^{-2} D_{z}(x, z) \tilde{p}_{z}(x, z, t),
\end{aligned}
$$

for all $(x, z) \in \bar{M}^{\prime}$ and $t \in[0, \infty)$. Here $\tilde{p}: \bar{M}^{\prime} \times[0, \infty) \mapsto \mathbb{R}$ is given by (2.46), (2.47) and $\hat{u}, \hat{w}, \hat{p}: \bar{M}^{\prime} \mapsto \mathbb{R}$ is that solution to $[\mathrm{SSP}]$ which satisfies (2.40). Moreover,

$$
\bar{p}(x, z, t) \rightarrow \hat{p}(x, z), \quad u(x, z, t) \rightarrow \hat{u}(x, z), \quad w(x, z, t) \rightarrow \hat{w}(x, z) \quad \text { as } t \rightarrow \infty
$$


uniformly for $(x, z) \in \bar{M}^{\prime}$.

We remark that since $\hat{p} \in C\left(\bar{M}^{\prime}\right) \cap P C^{1}\left(\bar{M}^{\prime}\right)$ and the initial data for [IBVP] $\bar{p}_{0} \in C\left(\bar{M}^{\prime}\right) \cap P C^{1}\left(\bar{M}^{\prime}\right)$, then Theorem 2.3 implies global asymptotic stability (up to the addition of a constant to $\hat{p})$ for $[\mathrm{SSP}]$ with respect to perturbations in $C\left(\bar{M}^{\prime}\right) \cap$ $P C^{1}\left(\bar{M}^{\prime}\right)$.

To complete the solution to the problem we are required to determine $\lambda_{n}(\epsilon)(>0)$ and its corresponding eigenfunction $\phi_{n}: \bar{M}^{\prime} \mapsto \mathbb{R}$ for each $n=1,2,3, \ldots$, together with the steady state $\hat{p}, \hat{u}, \hat{w}: \bar{M}^{\prime} \mapsto \mathbb{R}$ which satisfies the constraint (2.40). In the next sections we focus attention on the study of [SSP] and [EVP] in turn.

In particular for a thin porous layer the parameter $\epsilon$, which measures the aspect ratio of the layer, is small, so that $0<\epsilon \ll 1$. In the next two sections we will consider the structure of the solutions to [SSP] and [EVP] in the asymptotic limit $\epsilon \rightarrow 0$, via the method of matched asymptotic expansions.

3. Asymptotic solution to the steady state problem [SSP] as $\epsilon \rightarrow 0$. In this section we develop the uniform asymptotic structure of the solution to the steady state problem [SSP] (given by (2.23)-(2.29)) in the limit $\epsilon \rightarrow 0$, via the method of matched asymptotic expansions. We recall that existence and uniqueness, for each $\epsilon>0$, follows from Theorem 2.2, and following Theorem 2.3, we require the solution of [SSP] that satisfies the constraint (2.40). Due to the initial scalings in the nondimensionalization (2.12), we anticipate that $\hat{u}, \hat{w}, \hat{p}: \bar{M}^{\prime} \mapsto \mathbb{R}$ are such that

$$
\hat{u}, \hat{w}, \hat{p}=O(1)
$$

as $\epsilon \rightarrow 0$, uniformly for $(x, z) \in \bar{M}^{\prime} \backslash \bigcup_{i=1}^{N} \delta_{i}^{\epsilon}=\bar{N}_{\epsilon}^{\prime}$, where $\delta_{i}^{\epsilon}$ is an $\mathrm{O}(\epsilon)$ neighborhood of $\bar{d}_{i}$, for each $i=1, \ldots, N$. Therefore, following (3.1), we introduce the outer region (this being $\bar{N}_{\epsilon}^{\prime}$ ) asymptotic expansions

$$
\begin{aligned}
\hat{u}(x, z ; \epsilon) & =\hat{u}_{0}(x, z)+\epsilon \hat{u}_{1}(x, z)+O\left(\epsilon^{2}\right), \\
\hat{w}(x, z ; \epsilon) & =\hat{w}_{0}(x, z)+\epsilon \hat{w}_{1}(x, z)+O\left(\epsilon^{2}\right), \\
\hat{p}(x, z ; \epsilon) & =\hat{p}_{0}(x, z)+\epsilon \hat{p}_{1}(x, z)+O\left(\epsilon^{2}\right),
\end{aligned}
$$

as $\epsilon \rightarrow 0$, uniformly for $(x, z) \in \bar{N}_{\epsilon}^{\prime}$. We substitute from (3.2) into [SSP] and condition (2.40). At leading order we obtain the following problem for $\hat{u}_{0}, \hat{w}_{0}, \hat{p}_{0}: \bar{M}^{\prime} \mapsto \mathbb{R}$ :

$$
\begin{gathered}
\hat{u}_{0 x}+\hat{w}_{0 z}=\sum_{i=1}^{N} s_{i}(z) \delta\left(x-x_{i}\right), \quad(x, z) \in M^{\prime}, \\
\hat{u}_{0}=-D_{x}(x, z) \hat{p}_{0}, \quad(x, z) \in M^{\prime}, \\
0=-D_{z}(x, z) \hat{p_{0}}, \quad(x, z) \in M^{\prime}, \\
\hat{u}_{0}(-1, z)=0, \quad z \in\left[z_{-}(-1), z_{+}(-1)\right], \\
\hat{u}_{0}(1, z)=0, \quad z \in\left[z_{-}(1), z_{+}(1)\right], \\
\hat{w}_{0}\left(x, z_{+}(x)\right)-z_{+}^{\prime}(x) \hat{u}_{0}\left(x, z_{+}(x)\right)=0, \quad x \in(-1,1), \\
\hat{w}_{0}\left(x, z_{-}(x)\right)-z_{-}^{\prime}(x) \hat{u}_{0}\left(x, z_{-}(x)\right)=0, \quad x \in(-1,1), \\
\iint_{\bar{M}^{\prime}} \hat{p}_{0}(x, z) \mathrm{d} x \mathrm{~d} z=I_{0} .
\end{gathered}
$$

We now construct the solution of (3.3)-(3.10). As a consequence of (2.1), equation (3.5) requires

$$
\hat{p}_{0}(x, z)=A(x), \quad(x, z) \in \bar{M}^{\prime},
$$


with $A:[-1,1] \mapsto \mathbb{R}$ to be determined. Equation (3.4) then gives

$$
\hat{u}_{0}(x, z)=-D_{x}(x, z) A^{\prime}(x), \quad(x, z) \in \bar{M}^{\prime},
$$

and the boundary conditions (3.6) and (3.7) then require $A^{\prime}(-1)=A^{\prime}(1)=0$. We next substitute from (3.12) into (3.3), which becomes

$$
\hat{w}_{0 z}=\sum_{i=1}^{N} s_{i}(z) \delta\left(x-x_{i}\right)+\left[D_{x}(x, z) A^{\prime}(x)\right]_{x}, \quad(x, z) \in M^{\prime} .
$$

A direct integration of (3.13), together with an application of (3.9), leads to

$$
\begin{aligned}
& \hat{w}_{0}(x, z)=\sum_{i=1}^{N} F_{i}(z) \delta\left(x-x_{i}\right) \\
& \quad+\int_{z_{-}(x)}^{z}\left[D_{x}(x, \lambda) A^{\prime}(x)\right]_{x} \mathrm{~d} \lambda-z_{-}^{\prime}(x) D_{x}\left(x, z_{-}(x)\right) A^{\prime}(x), \quad(x, z) \in \bar{M}^{\prime},
\end{aligned}
$$

where

$$
F_{i}(z)=\int_{z_{-}\left(x_{i}\right)}^{z} s_{i}(\lambda) \mathrm{d} \lambda, \quad z \in\left[z_{-}\left(x_{i}\right), z_{+}\left(x_{i}\right)\right]
$$

for each $i=1, \ldots, N$. (Note that $F_{i}:\left[z_{-}\left(x_{i}\right), z_{+}\left(x_{i}\right)\right] \mapsto \mathbb{R}$ is such that $F_{i} \in$ $C^{1}\left(\left[z_{-}\left(x_{i}\right), z_{+}\left(x_{i}\right)\right]\right)$, for each $i=1, \ldots, N$.) It remains to apply the boundary condition (3.8). The application of (3.8) using (3.12) and (3.15) finally requires that

$$
\begin{gathered}
\int_{z_{-}(x)}^{z_{+}(x)}\left[D_{x}(x, \lambda) A^{\prime}(x)\right]_{x} \mathrm{~d} \lambda+\left\{z_{+}^{\prime}(x) D_{x}\left(x, z_{+}(x)\right)-z_{-}^{\prime}(x) D_{x}\left(x, z_{-}(x)\right)\right\} A^{\prime}(x) \\
+\sum_{i=1}^{N} \alpha_{i} \delta\left(x-x_{i}\right)=0, \quad x \in(-1,1) .
\end{gathered}
$$

We now rewrite the first term on the left-hand side of (3.16) as

$$
\begin{aligned}
& \int_{z_{-}(x)}^{z_{+}(x)}\left[D_{x}(x, \lambda) A^{\prime}(x)\right]_{x} \mathrm{~d} \lambda \\
& =\left(\int_{z_{-}(x)}^{z_{+}(x)} D_{x}(x, \lambda) A^{\prime}(x) \mathrm{d} \lambda\right)^{\prime}-\left\{z_{+}^{\prime}(x) D_{x}\left(x, z_{+}(x)\right)-z_{-}^{\prime}(x) D_{x}\left(x, z_{-}(x)\right)\right\} A^{\prime}(x) \\
& =\left(\bar{D}_{x}(x) A^{\prime}(x)\right)^{\prime}-\left\{z_{+}^{\prime}(x) D_{x}\left(x, z_{+}(x)\right)-z_{-}^{\prime}(x) D_{x}\left(x, z_{-}(x)\right)\right\} A^{\prime}(x), \quad x \in(-1,1) .
\end{aligned}
$$

On substitution from (3.17) into (3.16) we obtain

$$
\left(\bar{D}_{x}(x) A^{\prime}(x)\right)^{\prime}=-\sum_{i=1}^{N} \alpha_{i} \delta\left(x-x_{i}\right), \quad x \in(-1,1),
$$

with

$$
\bar{D}_{x}(x)=\int_{z_{-}(x)}^{z_{+}(x)} D_{x}(x, \lambda) \mathrm{d} \lambda, \quad x \in[-1,1],
$$


which represents the depth integrated permeability of the layer in the $x$-direction at each location $x \in[-1,1]$. We observe that $\bar{D}_{x}:[-1,1] \mapsto \mathbb{R}$ is such that $\bar{D}_{x} \in$ $C^{1}([-1,1])$ and $\bar{D}_{x}(x) \geq \bar{D}_{0}>0$ for all $x \in[-1,1]$, via $(2.1)$ and $(2.2)$, for some positive constant $\bar{D}_{0}$. Thus $A:[-1,1] \mapsto \mathbb{R}$ is determined as the solution to the linear, inhomogeneous, boundary value problem (hereafter referred to as [BVP]),

$$
\begin{gathered}
{\left[\bar{D}_{x}(x) A^{\prime}(x)\right]^{\prime}=-\sum_{i=1}^{N} \alpha_{i} \delta\left(x-x_{i}\right), \quad x \in(-1,1),} \\
A^{\prime}(-1)=A^{\prime}(1)=0, \\
\int_{-1}^{1}\left(z_{+}(x)-z_{-}(x)\right) A(x) \mathrm{d} x=I_{0},
\end{gathered}
$$

with the final constraint arising via (3.10) on using (3.11). We observe the following.

REMARK 3.1. [BVP] has a unique solution if and only if $\sum_{i=1}^{N} \alpha_{i}=0$.

This is in accord with condition (2.31) of Theorem 2.2. We now construct the solution to [BVP] (under condition (2.31)). It is straightforward to establish that the solution to [BVP] (with the usual Dirac delta function formalism) is given by

$$
A(x)=\int_{-1}^{x} \frac{S(\lambda)}{\bar{D}_{x}(\lambda)} \mathrm{d} \lambda+A_{0}, \quad x \in[-1,1]
$$

where here the function $S:[-1,1] \mapsto \mathbb{R}$ is the step function, given by

$$
S(\lambda)=-\sum_{i=0}^{k} \alpha_{i} \quad \text { for all } \lambda \in\left[x_{k}, x_{k+1}\right),
$$

and for each $k=0, \ldots, N$, where we have defined $\alpha_{0}=0, x_{0}=-1, x_{N+1}=1$. The constant $A_{0} \in \mathbb{R}$ is given by

$$
A_{0}=\frac{I_{0}}{\operatorname{meas}\left(\bar{M}^{\prime}\right)}-\frac{1}{\operatorname{meas}\left(\bar{M}^{\prime}\right)} \int_{-1}^{1} \frac{S(\lambda) \operatorname{meas}\left(\bar{M}^{\prime}(\lambda)\right)}{\bar{D}_{x}(\lambda)} \mathrm{d} \lambda
$$

where

$$
\operatorname{meas}\left(\bar{M}^{\prime}(\lambda)\right)=\int_{\lambda}^{1}\left(z_{+}(\mu)-z_{-}(\mu)\right) \mathrm{d} \mu \quad \text { for all } \lambda \in[-1,1]
$$

so that meas $\left(\bar{M}^{\prime}(-1)\right)=$ meas $\left(\bar{M}^{\prime}\right)$. We observe that $A \in C([-1,1]) \cap P C^{2}([-1,1])$, and that $A^{\prime}\left(x_{j}^{+}\right)-A^{\prime}\left(x_{j}^{-}\right)=-\alpha_{j} / \bar{D}_{x}\left(x_{j}\right)$ for each $j=1, \ldots, N$. We can now reconstruct the solution to the leading order problem as

$$
\begin{gathered}
\hat{p}_{0}(x, z)=A(x), \quad(x, z) \in \bar{M}^{\prime}, \\
\hat{u}_{0}(x, z)=\frac{-D_{x}(x, z)}{\bar{D}_{x}(x)} S(x), \quad(x, z) \in \bar{M}^{\prime}
\end{gathered}
$$

$$
\hat{w}_{0}(x, z)=S(x) \int_{z_{-}(x)}^{z}\left\{\frac{D_{x}(x, \lambda)}{\bar{D}_{x}(x)}\right\}_{x} \mathrm{~d} \lambda-\frac{z_{-}^{\prime}(x) D_{x}\left(x, z_{-}(x)\right) S(x)}{\bar{D}_{x}(x)}, \quad(x, z) \in \bar{N}_{\epsilon}^{\prime},
$$


from (3.11), (3.12), (3.14), and (3.19). It follows from (3.22)-(3.24) that $\hat{p}_{0} \in C\left(\bar{M}^{\prime}\right) \cap$ $C^{1}\left(\bar{M}^{\prime} \backslash \bar{d}\right) \cap C^{2}\left(M^{\prime} \backslash d\right), \hat{u}_{0} \in C\left(\bar{M}^{\prime} \backslash \bar{d}\right) \cap C^{1}\left(M^{\prime} \backslash d\right), \hat{w}_{0} \in C\left(\bar{M}^{\prime} \backslash \bar{d}\right) \cap C^{1}\left(M^{\prime} \backslash d\right)$, and

$$
\begin{gathered}
{\left[\hat{p}_{0} z\right]_{x_{i}^{-}}^{x_{i}^{+}}=0,} \\
{\left[\hat{p}_{0 x}\right]_{x_{i}^{-}}^{x_{i}^{+}}=\frac{-\alpha_{i}}{\bar{D}_{x}\left(x_{i}\right)},} \\
{\left[\hat{u}_{0}\right]_{x_{i}^{-}}^{x_{i}^{+}}=\frac{D_{x}\left(x_{i}, z\right) \alpha_{i}}{\bar{D}_{x}\left(x_{i}\right)},} \\
{\left[\hat{w}_{0}\right]_{x_{i}^{-}}^{x^{+}}=\left\{\frac{z_{-}^{\prime}\left(x_{i}\right) D_{x}\left(x_{i}, z_{-}\left(x_{i}\right)\right)}{\bar{D}_{x}\left(x_{i}\right)}-\int_{z_{-}\left(x_{i}\right)}^{z}\left[\left\{\frac{D_{x}(x, \lambda)}{\bar{D}_{x}(x)}\right\}_{x}\right]_{x=x_{i}} \mathrm{~d} \lambda\right\} \alpha_{i}}
\end{gathered}
$$

for $z \in\left[z_{-}\left(x_{i}\right), z_{+}\left(x_{i}\right)\right]$ and for each $i=1, \ldots, N$. We now proceed to $O(\epsilon)$. The problem for $\hat{u}_{1}, \hat{w}_{1}, \hat{p}_{1}: \bar{M}^{\prime} \mapsto \mathbb{R}$ is similar to the leading order problem and is not repeated here. We obtain

$$
\begin{gathered}
\hat{p}_{1}(x, z)=B(x), \quad(x, z) \in \bar{M}^{\prime} \\
\hat{u}_{1}(x, z)=-D_{x}(x, z) B^{\prime}(x), \quad(x, z) \in \bar{M}^{\prime} \\
\hat{w}_{1}(x, z)=\int_{z_{-}(x)}^{z}\left[D_{x}(x, \lambda) B^{\prime}(x)\right]_{x} \mathrm{~d} \lambda-z_{-}^{\prime}(x) D_{x}\left(x, z_{-}(x)\right) B^{\prime}(x), \quad(x, z) \in \bar{M}^{\prime},
\end{gathered}
$$

where $B:[-1,1] \mapsto \mathbb{R}$ is the solution to the boundary value problem

$$
\begin{gathered}
{\left[\bar{D}_{x}(x) B^{\prime}(x)\right]^{\prime}=0, \quad x \in(-1,1),} \\
B^{\prime}(-1)=B^{\prime}(1)=0 \\
\int_{-1}^{1}\left(z_{+}(x)-z_{-}(x)\right) B(x) \mathrm{d} x=0 .
\end{gathered}
$$

The unique solution $B \in C^{1}([-1,1]) \cap C^{2}((-1,1))$ is given by $B(x)=0$ for all $x \in[-1,1]$, and so $\hat{p}_{1}(x, z)=\hat{u}_{1}(x, z)=\hat{w}_{1}(x, z)=0$ for $(x, z) \in \bar{M}^{\prime}$, via (3.29). The outer region asymptotic expansions are thus

$$
\begin{gathered}
\hat{u}(x, z ; \epsilon)=\frac{-D_{x}(x, z)}{\bar{D}_{x}(x)} S(x)+O\left(\epsilon^{2}\right) \\
\hat{w}(x, z ; \epsilon)=S(x) \int_{z_{-}(x)}^{z}\left\{\frac{D_{x}(x, \lambda)}{\bar{D}_{x}(x)}\right\}_{x} \mathrm{~d} \lambda-\frac{z_{-}^{\prime}(x) D_{x}\left(x, z_{-}(x)\right) S(x)}{\bar{D}_{x}(x)}+O\left(\epsilon^{2}\right), \\
\hat{p}(x, z ; \epsilon)=A(x)+O\left(\epsilon^{2}\right),
\end{gathered}
$$

as $\epsilon \rightarrow 0$, uniformly for $(x, z) \in \bar{N}_{\epsilon}^{\prime}$, with $A, S:[-1,1] \mapsto \mathbb{R}$ given by (3.19)-(3.21).

We now observe from (3.30)-(3.32), via (3.25)-(3.28), that all of the regularity requirements in (si), together with the limit conditions (sii), (siii), and (2.30), are not satisfied at $x=x_{i}$ for each $z \in\left[z_{-}\left(x_{i}\right), z_{+}\left(x_{i}\right)\right]$, with $i=1, \ldots, N$ (although the integrated forms are satisfied). We conclude (as was anticipated earlier) that the outer region asymptotic expansions (3.30)-(3.32) become nonuniform when $(x, z) \in \delta_{i}^{\epsilon}$ as $\epsilon \rightarrow 0, i=1, \ldots, N$. To obtain a uniform asymptotic representation to the solution to [SSP] when $(x, z) \in \delta_{i}^{\epsilon}$ as $\epsilon \rightarrow 0$, we must therefore introduce an inner region at each line source/sink location $x=x_{i}, i=1, \ldots, N$. We now consider the inner region in the neighborhood of $x=x_{i}$ in detail. In the inner region, $x=x_{i}+O(\epsilon), z=O(1)$, 
as $\epsilon \rightarrow 0$, with, from (3.30)-(3.32), $\hat{u}=O(1), \hat{w}=O\left(\epsilon^{-1}\right), \hat{p}=A_{i}+O(\epsilon)$, as $\epsilon \rightarrow 0$, with $A_{i}=A\left(x_{i}\right), i=1, \ldots, N$. Thus, in the inner region we write

$$
x=x_{i}+\epsilon X,
$$

with $X \in(-\infty, \infty)$ such that $X=O(1)$ as $\epsilon \rightarrow 0$, together with

$$
\hat{u}=U, \quad \hat{w}=\epsilon^{-1} W, \quad \hat{p}=A_{i}+\epsilon P,
$$

where $U, W, P:(-\infty, \infty) \times\left[z_{-}\left(x_{i}\right), z_{+}\left(x_{i}\right)\right] \mapsto \mathbb{R}$ are such that $U, W, P=O(1)$ as $\epsilon \rightarrow 0$. We now substitute from (3.33), (3.34) into the full problem [SSP] ((2.23), (2.24), (2.25), (2.28), (2.29), excluding conditions (2.26), (2.27) which lie outside the inner region in the limit $\epsilon \rightarrow 0$ ). The full problem in the inner region then becomes

$$
\begin{gathered}
U_{X}+W_{z}=s_{i}(z) \delta(X), \quad(X, z) \in D(\epsilon), \\
U=-D_{x}\left(x_{i}+\epsilon X, z\right) P_{X}, \quad(X, z) \in D(\epsilon), \\
W=-D_{z}\left(x_{i}+\epsilon X, z\right) P_{z}, \quad(X, z) \in D(\epsilon), \\
W-\epsilon z_{+}^{\prime}\left(x_{i}+\epsilon X\right) U=0, \quad X \in(-\infty, \infty), \quad z=z_{+}\left(x_{i}+\epsilon X\right), \\
W-\epsilon z_{-}^{\prime}\left(x_{i}+\epsilon X\right) U=0, \quad X \in(-\infty, \infty), \quad z=z_{-}\left(x_{i}+\epsilon X\right),
\end{gathered}
$$

together with matching conditions to the outer region as $|X| \rightarrow \infty$. Here

$$
D(\epsilon)=\left\{(X, z) \in \mathbb{R}^{2}: X \in(-\infty, \infty) \text { and } z \in\left(z_{-}\left(x_{i}+\epsilon X\right), z_{+}\left(x_{i}+\epsilon X\right)\right)\right\} .
$$

We now introduce the inner region asymptotic expansions as

$$
\begin{aligned}
U(X, z ; \epsilon) & =U_{0}(X, z)+O(\epsilon), \\
W(X, z ; \epsilon) & =W_{0}(X, z)+O(\epsilon), \\
P(X, z ; \epsilon) & =P_{0}(X, z)+O(\epsilon),
\end{aligned}
$$

as $\epsilon \rightarrow 0$, with $(X, z) \in \bar{D}(\epsilon)$. On substitution from (3.41) into (3.35)-(3.40) we obtain the leading order problem as

$$
\begin{gathered}
U_{0 X}+W_{0 z}=s_{i}(z) \delta(X), \quad(X, z) \in D(0), \\
U_{0}=-\tilde{D}_{x}(z) P_{0 X}, \quad(X, z) \in D(0), \\
W_{0}=-\tilde{D}_{z}(z) P_{0 z}, \quad(X, z) \in D(0), \\
W_{0}\left(X, z_{+}^{i}\right)=0, \quad X \in(-\infty, \infty), \\
W_{0}\left(X, z_{-}^{i}\right)=0, \quad X \in(-\infty, \infty) .
\end{gathered}
$$

Here,

$$
\tilde{D}_{x}(z)=D_{x}\left(x_{i}, z\right), \quad \tilde{D}_{z}(z)=D_{z}\left(x_{i}, z\right),
$$

for all $z \in\left[z_{-}^{i}, z_{+}^{i}\right]$, with $z_{-}^{i}=z_{-}\left(x_{i}\right)$ and $z_{+}^{i}=z_{+}\left(x_{i}\right)$. Also, $\bar{D}(0)$, via (3.40), is now the unbounded region in the $(X, z)$ plane contained between the coordinate lines $z=z_{-}^{i}$ and $z=z_{+}^{i}$; that is,

$$
\bar{D}(0)=(-\infty, \infty) \times\left[z_{-}^{i}, z_{+}^{i}\right] .
$$

The leading order problem (3.42)-(3.46) is completed by applying the asymptotic matching principle of Van Dyke [20]. It is straightforward to establish that matching 
of $\hat{p}$ is sufficient, after which matching of $\hat{u}$ and $\hat{w}$ follows automatically. We must apply Van Dyke's matching principle to the outer region asymptotic expansion for $\hat{p}$ taken to $O(\epsilon),(3.32)$, with the inner region asymptotic expansion for $\hat{p}$ taken to $O(\epsilon)$, (3.34) and (3.41). The appropriate matching condition is readily determined as

$$
P_{0}(X, z)=A_{i}^{ \pm^{\prime}} X+o(1) \quad \text { as } X \rightarrow \pm \infty, \text { uniformly for } z \in\left[z_{-}^{i}, z_{+}^{i}\right]
$$

with

$$
{A_{i}^{+{ }^{\prime}}}^{\prime}=A^{\prime}\left(x_{i}^{+}\right)=-\frac{\sum_{j=0}^{i} \alpha_{j}}{\bar{D}_{x}\left(x_{i}\right)},{A_{i}^{{ }^{\prime}}}^{\prime}=A^{\prime}\left(x_{i}^{-}\right)=-\frac{\sum_{j=0}^{i-1} \alpha_{j}}{\bar{D}_{x}\left(x_{i}\right)} \text {, for } i=1, \ldots, N,
$$

via (3.19) and (3.20). Finally the regularity conditions (si)-(siii) with (2.30) require the following:

(Ii) $P_{0} \in C(\bar{D}(0)) \cap C^{1}(\bar{D}(0) \backslash \bar{I}) \cap C^{2}(D(0) \backslash I), U_{0} \in C(\bar{D}(0) \backslash \bar{I}) \cap C^{1}(D(0) \backslash I)$, $W_{0} \in C(\bar{D}(0)) \cap C^{1}(D(0) \backslash I)$, where $I=\{0\} \times\left(z_{-}^{i}, z_{+}^{i}\right)$;

(Iii) $\lim _{X \rightarrow 0^{ \pm}} P_{0 X}$ and $\lim _{X \rightarrow 0^{ \pm}} P_{0 z}$ both exist uniformly for $z \in\left[z_{-}^{i}, z_{+}^{i}\right]$;

(Iiii) $\left[P_{0 z}\right]_{0^{-}}^{0^{+}}=0,\left[P_{0 X}\right]_{0^{-}}^{0^{+}}=-s_{i}(z) / \tilde{D}_{x}(z),\left[U_{0}\right]_{0^{-}}^{0^{+}}=s_{i}(z)$, and $\left[W_{0}\right]_{0^{-}}^{0^{+}}=0$ for all $z \in\left[z_{-}^{i}, z_{+}^{i}\right]$.

We can now eliminate $U_{0}$ and $W_{0}$, via (3.43) and (3.44), and obtain the following strongly elliptic problem for $P_{0}$, namely,

$$
\begin{gathered}
\left(\tilde{D}_{x}(z) P_{0 X}\right)_{X}+\left(\tilde{D}_{z}(z) P_{0 z}\right)_{z}=-s_{i}(z) \delta(X), \quad(X, z) \in D(0), \\
P_{0 z}\left(X, z_{+}^{i}\right)=0, \quad X \in(-\infty, \infty), \\
P_{0 z}\left(X, z_{-}^{i}\right)=0, \quad X \in(-\infty, \infty), \\
P_{0}(X, z)=A_{i}^{ \pm^{\prime}} X+o(1), \quad X \rightarrow \pm \infty, \quad \text { uniformly for } z \in\left[z_{-}^{i}, z_{+}^{i}\right],
\end{gathered}
$$

together with (Ii)-(Iiii). The first step in obtaining the solution to (3.51)-(3.54) is to consider the regular Sturm-Liouville eigenvalue problem,

$$
\begin{gathered}
\left(\tilde{D}_{z}(z) \psi_{z}\right)_{z}+\bar{\lambda} \tilde{D}_{x}(z) \psi=0, \quad z \in\left(z_{-}^{i}, z_{+}^{i}\right), \\
\psi_{z}\left(z_{-}^{i}\right)=\psi_{z}\left(z_{+}^{i}\right)=0,
\end{gathered}
$$

which we refer to as $[\mathrm{SL}]$. Here $\bar{\lambda} \in \mathbb{C}$ is the eigenvalue parameter. Classical SturmLiouville theory (see, for example, [6, Chapters 7, 8]) determines that the set of eigenvalues of $[\mathrm{SL}]$ is given by $\bar{\lambda}=\bar{\lambda}_{r} \in \mathbb{R}, r=0,1,2, \ldots$, with $0=\bar{\lambda}_{0}<\bar{\lambda}_{1}<\bar{\lambda}_{2}<$ $\bar{\lambda}_{3}<\cdots$, where $\bar{\lambda}_{r} \rightarrow+\infty$ as $r \rightarrow \infty$. The corresponding normalized eigenfunctions $\psi_{r}:\left[z_{-}^{i}, z_{+}^{i}\right] \mapsto \mathbb{R}$ form an orthonormal set, so that

$$
\left\langle\psi_{r}, \psi_{s}\right\rangle=\int_{z_{-}^{i}}^{z_{+}^{i}} \tilde{D}_{x}(z) \psi_{r}(z) \psi_{s}(z) \mathrm{d} z=\delta_{r s} \quad \text { for } r, s=0,1,2, \ldots
$$

The set of eigenfunctions of $[\mathrm{SL}]$ are complete on the interval $\left[z_{-}^{i}, z_{+}^{i}\right]$. Completeness allows us to write the solution to (3.51) with conditions (3.52), (3.53) as

$$
P_{0}(X, z)= \begin{cases}\sum_{n=0}^{\infty} \chi_{n}^{+}(X) \psi_{n}(z), & X>0 \\ \sum_{n=0}^{\infty} \chi_{n}^{-}(X) \psi_{n}(z), & X<0\end{cases}
$$

with $z \in\left[z_{-}^{i}, z_{+}^{i}\right]$. Substitution of (3.56) into (3.51) establishes that

$$
\begin{array}{ll}
\chi_{n}^{+}(X)=A_{n} \mathrm{e}^{\bar{\lambda}_{n}^{1 / 2} X}+B_{n} \mathrm{e}^{-\bar{\lambda}_{n}^{1 / 2} X}, & X>0, \\
\chi_{n}^{-}(X)=D_{n} \mathrm{e}^{\bar{\lambda}_{n}^{1 / 2} X}+C_{n} \mathrm{e}^{-\bar{\lambda}_{n}^{1 / 2} X}, & X<0,
\end{array}
$$


with $A_{n}, B_{n}, C_{n}, D_{n} \in \mathbb{R}$ constants, for $n=1,2, \ldots$ With $n=0$, we have

$$
\begin{aligned}
& \chi_{0}^{+}(X)=A_{0}+B_{0} X, \quad X>0, \\
& \chi_{0}^{-}(X)=C_{0}+D_{0} X, \quad X<0,
\end{aligned}
$$

with $A_{0}, B_{0}, C_{0}, D_{0} \in \mathbb{R}$ constants. At this stage we observe that

$$
\psi_{0}(z)=\left\{\int_{z_{-}^{i}}^{z_{+}^{i}} \tilde{D}_{x}(s) \mathrm{d} s\right\}^{-1 / 2}=\Psi_{0}, \quad z \in\left[z_{-}^{i}, z_{+}^{i}\right],
$$

with $\Psi_{0}>0$. It then follows, via (3.56)-(3.59), that the boundary conditions (3.49) are satisfied if and only if

$$
\begin{array}{lll}
A_{n}=0, & n=0,1,2, \ldots, & B_{0}=A_{i}^{+^{\prime}} \Psi_{0}^{-1}, \\
C_{n}=0, & n=0,1,2, \ldots, & D_{0}=A_{i}^{-{ }^{\prime}} \Psi_{0}^{-1} .
\end{array}
$$

Next, across $X=0$, continuity of $P_{0}$, together with the condition $\left[P_{0}\right]_{0^{-}}^{0^{+}}=0$ of (Iiii), is satisfied if and only if, via (3.56)-(3.60),

$$
B_{n}=D_{n}, \quad n=1,2, \ldots
$$

Finally, it remains to satisfy the condition $\left[P_{0 X}\right]_{0^{-}}^{0^{+}}=-s_{i}(z) / \tilde{D}_{x}(z)$ of (Iiii), which requires, via (3.56)-(3.61), that

$$
\left(A_{i}^{+^{\prime}}-{A_{i}^{-{ }^{\prime}}}^{\prime}\right)-\sum_{n=1}^{\infty} 2 \bar{\lambda}_{n}^{1 / 2} B_{n} \psi_{n}(z)=\frac{-s_{i}(z)}{\tilde{D}_{x}(z)}, \quad z \in\left[z_{-}^{i}, z_{+}^{i}\right] .
$$

The completeness of the eigenfunctions $\psi_{n}(z)(n=0,1,2, \ldots)$ on the interval $\left[z_{-}^{i}, z_{+}^{i}\right]$ allows (3.62) to be satisfied uniquely, with, using (3.55),

$$
{A_{i}^{+}}^{\prime}-A_{i}^{-^{\prime}}=-\frac{\int_{z_{-}^{i}}^{z_{+}^{i}} s_{i}(s) \mathrm{d} s}{\int_{z_{-}^{i}}^{z_{+}^{i}} \tilde{D}_{x}(s) \mathrm{d} s}=-\frac{\alpha_{i}}{\bar{D}_{x}\left(x_{i}\right)}
$$

via (3.47) and (3.18), and which is automatically satisfied using (3.50), and

$$
B_{k}=\frac{1}{2 \bar{\lambda}_{k}^{1 / 2}} \int_{z_{-}^{i}}^{z_{+}^{i}} s_{i}(s) \psi_{k}(s) \mathrm{d} s, \quad k=1,2, \ldots .
$$

Thus, the solution to (3.51)-(3.54), with regularity (Ii)-(Iiii), is given by

$$
P_{0}(X, z)= \begin{cases}A_{i}^{{ }^{\prime}} X+\sum_{n=1}^{\infty} B_{n} \mathrm{e}^{-\bar{\lambda}_{n}^{1 / 2} X} \psi_{n}(z), & X>0, \\ A_{i}^{{ }^{\prime}} X+\sum_{n=1}^{\infty} B_{n} \mathrm{e}^{\bar{\lambda}_{n}^{1 / 2} X} \psi_{n}(z), & X<0,\end{cases}
$$

with $z \in\left[z_{-}^{i}, z_{+}^{i}\right]$, and the coefficients $B_{n}, n=1,2, \ldots$, given by (3.63). $U_{0}(X, z)$ and $W_{0}(X, z)$ are now obtained directly from (3.43) and (3.44) as

$$
\begin{aligned}
& U_{0}(X, z)= \begin{cases}-\tilde{D}_{x}(z)\left\{{A_{i}^{+}}^{\prime}-\sum_{n=1}^{\infty} \bar{\lambda}_{n}^{1 / 2} B_{n} \mathrm{e}^{-\bar{\lambda}_{n}^{1 / 2} X} \psi_{n}(z)\right\}, & X>0, \\
-\tilde{D}_{x}(z)\left\{A_{i}^{{ }^{\prime}}+\sum_{n=1}^{\infty} \bar{\lambda}_{n}^{1 / 2} B_{n} \mathrm{e}^{\bar{\lambda}_{n}^{1 / 2} X} \psi_{n}(z)\right\}, & X<0,\end{cases} \\
& W_{0}(X, z)= \begin{cases}-\tilde{D}_{z}(z) \sum_{n=1}^{\infty} B_{n} \mathrm{e}^{-\bar{\lambda}_{n}^{1 / 2} X} \psi_{n}^{\prime}(z), & X>0, \\
-\tilde{D}_{z}(z) \sum_{n=1}^{\infty} B_{n} \mathrm{e}^{\bar{\lambda}_{n}^{1 / 2} X} \psi_{n}^{\prime}(z), & X<0,\end{cases}
\end{aligned}
$$


with $z \in\left[z_{-}^{i}, z_{+}^{i}\right]$. The only remaining question is how to actually compute the eigenvalues and corresponding eigenfunctions of [SL]. If $\tilde{D}_{x}, \tilde{D}_{z}$, are constant with respect to $z$, then analytical solution of [SL] is trivial. More generally, since [SL] is a regular Sturm-Liouville problem, numerical methods are straightforward and very efficient and will not be discussed further here. The solution of the leading order problem is now complete.

It is of interest to obtain the expression for the pressure at the location of the $i$ th line source/sink. From (3.34) and (3.41), this is given by $\hat{p}\left(x_{i}, z\right)=A_{i}+\epsilon P_{0}(0, z)+$ $O\left(\epsilon^{2}\right)$, for $z \in\left[z_{-}^{i}, z_{+}^{i}\right]$. On using (3.64), this becomes

$$
\hat{p}\left(x_{i}, z\right)=A_{i}+\epsilon\left(\sum_{n=1}^{\infty} B_{n} \psi_{n}(z)\right)+O\left(\epsilon^{2}\right) \quad \text { for } z \in\left[z_{-}^{i}, z_{+}^{i}\right] .
$$

The pressure difference between the $i$ th and $j$ th line source/sinks is then

$$
\begin{aligned}
\Delta \hat{p}_{i j}(z) & =\hat{p}\left(x_{i}, z\right)-\hat{p}\left(x_{j}, z\right) \\
& =\left(A_{i}-A_{j}\right)+\epsilon\left(\sum_{n=1}^{\infty}\left[B_{n}^{i} \psi_{n}^{i}(z)-B_{n}^{j} \psi_{n}^{j}(z)\right]\right)+O\left(\epsilon^{2}\right)
\end{aligned}
$$

for $z \in\left[z_{-}^{i}, z_{+}^{i}\right]$, with superscripts $i$ and $j$ distinguishing evaluation at the $i$ th and $j$ th line source/sink, respectively. In (3.68), we recall that, via (3.19),

$$
A_{i}-A_{j}=A\left(x_{i}\right)-A\left(x_{j}\right)=\int_{x_{j}}^{x_{i}} \frac{S(\lambda)}{\bar{D}_{x}(\lambda)} \mathrm{d} \lambda .
$$

The asymptotic structure to the solution of [SSP] as $\epsilon \rightarrow 0$ is now complete. Two minor extensions are worthy of consideration at this stage and are given in the subsections that follow.

3.1. A line source/sink close to the boundary. In the above, the locations of the line source/sinks $x_{i} \in(-1,1), i=1, \ldots, N$, are such that $\left(x_{1}+1\right),\left(1-x_{N}\right)$, and $x_{i+1}-x_{i}(i=1, \ldots, N-1)$ remain positive and finite $(O(1))$ as $\epsilon \rightarrow 0$. In this extension we consider the situation when $x_{1}+1=O(\epsilon)$ as $\epsilon \rightarrow 0$, so that the line source/sink at $x=x_{1}$ lies within $O(\epsilon)$ of the layer boundary at $x=-1$. The structure of the outer region to $[\mathrm{SSP}]$ is unchanged. However, the inner region to [SSP] at $x=x_{1}$ now encompasses the boundary at $x=-1$, and so the leading order problem in this inner region, when $i=1$, is modified. To formalize this we write

$$
x_{1}=-1+\epsilon \bar{\sigma},
$$

with the constant $\bar{\sigma}>0$. In terms of the inner coordinate $X$,

$$
x=x_{1}+\epsilon X,
$$

via (3.33). Thus, via (3.69) and (3.70), in the inner region, the line source/sink is located at $X=0$, while the layer boundary is located at $X=-\bar{\sigma}$. Without repeating details, the leading order problem in the inner region is now

$$
\begin{gathered}
U_{0 X}+W_{0 z}=s_{1}(z) \delta(X), \quad(X, z) \in D(0), \\
U_{0}=-\tilde{D}_{x}(z) P_{0 X}, \quad(X, z) \in D(0), \\
W_{0}=-\tilde{D}_{z}(z) P_{0 z}, \quad(X, z) \in D(0), \\
W_{0}\left(X, z_{+}^{1}\right)=0, \quad X \in(-\bar{\sigma}, \infty), \\
W_{0}\left(X, z_{-}^{1}\right)=0, \quad X \in(-\bar{\sigma}, \infty),
\end{gathered}
$$


where now $\bar{D}(0)$ is the unbounded region in the $(X, z)$ plane contained inside the coordinate lines $z=z_{+}^{1}, z=z_{-}^{1}$, and $X=-\bar{\sigma}$, so that

$$
\bar{D}(0)=[-\bar{\sigma}, \infty) \times\left[z_{-}^{1}, z_{+}^{1}\right] .
$$

The leading order problem (3.71)-(3.75) is completed with the condition

$$
\begin{gathered}
U_{0}(-\bar{\sigma}, z)=0, \quad z \in\left[z_{-}^{1}, z_{+}^{1}\right], \\
P_{0}(X, z)={A_{1}^{+}}^{\prime} X+o(1), \text { as } X \rightarrow+\infty, \text { uniformly for } z \in\left[z_{-}^{1}, z_{+}^{1}\right],
\end{gathered}
$$

with the latter being the matching condition to the outer region. The solution to (3.71)-(3.78) can be constructed as before, to obtain

$$
P_{0}(X, z)= \begin{cases}{A_{1}^{+}}^{\prime} X+\sum_{n=1}^{\infty} \bar{B}_{n} \mathrm{e}^{-\bar{\lambda}_{n}^{1 / 2} X} \psi_{n}(z), & X>0, \\ \sum_{n=1}^{\infty} \frac{\bar{B}_{n}}{\cosh \left[\bar{\lambda}_{n}^{1 / 2}(X+\bar{\sigma})\right]} \cosh \bar{\lambda}_{n}^{1 / 2}(X+\bar{\sigma}) \psi_{n}(z), & -\bar{\sigma} \leq X<0,\end{cases}
$$

for $z \in\left[z_{-}^{1}, z_{+}^{1}\right]$, and with

$$
\bar{B}_{n}=\frac{1}{\bar{\lambda}_{n}^{1 / 2}\left(1+\tanh \left(\bar{\lambda}_{n}^{1 / 2} \bar{\sigma}\right)\right)} \int_{z_{-}^{1}}^{z_{+}^{1}} s_{1}(s) \psi_{n}(s) \mathrm{d} s, \quad n=1,2, \ldots
$$

Equations (3.72)-(3.73) then give the corresponding expressions for $U_{0}(X, z)$ and $W_{0}(X, z)$. The pressure at the location of this first line source/sink is then given by

$$
\hat{p}\left(x_{1}, z\right)=A_{1}+\epsilon\left(\sum_{n=1}^{\infty} \bar{B}_{n} \psi_{n}(z)\right)+O\left(\epsilon^{2}\right)
$$

for $z \in\left[z_{-}^{1}, z_{+}^{1}\right]$, with $\bar{B}_{n}, n=1,2, \ldots$, as given in (3.79). The difference in the expression for pressure at the wall close line source/sink (3.80), and at the interior line source/sink (3.67), occurs in the expressions for the sequence of constants $B_{n}$, (3.63), and $\bar{B}_{n},(3.79), n=1,2, \ldots$

3.2. Two closely located line source/sinks. In this extension, we consider the situation when the $k$ th and $(k+1)$ th line source/sinks are within $O(\epsilon)$ separation of each other. With $x_{k}, x_{k+1} \in(-1,1)$, we write $x_{k+1}=x_{k}+\tilde{\sigma} \epsilon$, with the constant $\tilde{\sigma}>0$. In terms of the inner coordinate $X, x=x_{k}+\epsilon X$, via (3.33). Thus both line source/sinks at $x=x_{k}$ and $x=x_{k+1}$ are located in the inner region at $x=x_{k}$, with their respective locations in this inner region being at $X=0$ and $X=\tilde{\sigma}$. Without repeating details, the leading order problem in the inner region is now

$$
\begin{gathered}
U_{0 X}+W_{0 z}=s_{k}(z) \delta(X)+s_{k+1}(z) \delta(X-\tilde{\sigma}), \quad(X, z) \in D(0), \\
U_{0}=-\tilde{D}_{x}(z) P_{0 X}, \quad(X, z) \in D(0), \\
W_{0}=-\tilde{D}_{z}(z) P_{0 z}, \quad(X, z) \in D(0), \\
W_{0}\left(X, z_{+}^{k}\right)=0, \quad X \in(-\infty, \infty), \\
W_{0}\left(X, z_{-}^{k}\right)=0, \quad X \in(-\infty, \infty), \\
P_{0}(X, z)= \begin{cases}A_{k+1}^{+}{ }^{\prime}(X-\tilde{\sigma})+\tilde{\sigma} A_{k+1}^{-}{ }^{\prime}+o(1), & X \rightarrow+\infty, \\
A_{k}^{-\prime} X+o(1), & X \rightarrow-\infty,\end{cases}
\end{gathered}
$$


with $\bar{D}(0)$ as in (3.48) when $i=k$, and condition (3.86) being the appropriate matching condition to the outer region. The solution to (3.81)-(3.86) can be constructed as before, to obtain

$P_{0}(X, z)= \begin{cases}A_{k}^{-\prime} X+\sum_{n=1}^{\infty} \hat{B}_{n} \mathrm{e}^{\bar{\lambda}_{n}^{1 / 2} X} \psi_{n}(z), & X<0, \\ A_{k+1}^{-}{ }^{\prime} X+\sum_{n=1}^{\infty}\left(\hat{B}_{n} \cosh \left(\bar{\lambda}_{n}^{1 / 2} X\right)+\hat{D}_{n} \sinh \left(\bar{\lambda}_{n}^{1 / 2} X\right)\right) \psi_{n}(z), & 0<X<\tilde{\sigma}, \\ \left\{\tilde{\sigma} A_{k+1}^{-}{ }^{\prime}+A_{k+1}^{+}{ }^{\prime}(X-\tilde{\sigma})\right\}+\sum_{n=1}^{\infty} \hat{C}_{n} \mathrm{e}^{-\bar{\lambda}_{n}^{1 / 2}(X-\tilde{\sigma})} \psi_{n}(z), & X>\tilde{\sigma},\end{cases}$

with $z \in\left[z_{-}^{k}, z_{+}^{k}\right]$, and the coefficients

$\hat{B}_{n}=\frac{1}{2 \bar{\lambda}_{n}^{1 / 2}}\left\{\frac{1}{\left[\cosh \left(\bar{\lambda}_{n}^{1 / 2} \tilde{\sigma}\right)+\sinh \left(\bar{\lambda}_{n}^{1 / 2} \tilde{\sigma}\right)\right]} \int_{z_{-}^{k}}^{z_{+}^{k}} s_{k+1}(s) \psi_{n}(s) \mathrm{d} s+\int_{z_{-}^{k}}^{z_{+}^{k}} s_{k}(s) \psi_{n}(s) \mathrm{d} s\right\}$,

$\hat{D}_{n}=\frac{1}{2 \bar{\lambda}_{n}^{1 / 2}}\left\{\frac{1}{\left[\cosh \left(\bar{\lambda}_{n}^{1 / 2} \tilde{\sigma}\right)+\sinh \left(\bar{\lambda}_{n}^{1 / 2} \tilde{\sigma}\right)\right]} \int_{z_{-}^{k}}^{z_{+}^{k}} s_{k+1}(s) \psi_{n}(s) \mathrm{d} s-\int_{z_{-}^{k}}^{z_{+}^{k}} s_{k}(s) \psi_{n}(s) \mathrm{d} s\right\}$,
$\hat{C}_{n}=\frac{1}{2 \bar{\lambda}_{n}^{1 / 2}}\left\{\int_{z_{-}^{k}}^{z_{+}^{k}} s_{k+1}(s) \psi_{n}(s) \mathrm{d} s+\left[\cosh \left(\bar{\lambda}_{n}^{1 / 2} \tilde{\sigma}\right)-\sinh \left(\bar{\lambda}_{n}^{1 / 2} \tilde{\sigma}\right)\right] \int_{z_{-}^{k}}^{z_{+}^{k}} s_{k}(s) \psi_{n}(s) \mathrm{d} s\right\}$.

Equations (3.82)-(3.83) then give the corresponding expressions for $W_{0}(X, z)$ and $U_{0}(X, z)$. (Note that $\tilde{D}_{x}(z)$ and $\tilde{D}_{z}(z)$ in $(3.82),(3.83)$ are evaluated at $x=x_{k}$.) The pressures at the line source/sinks at $x=x_{k}$ and $x=x_{k+1}$ are given by, via (3.87),

$$
\begin{gathered}
\hat{p}\left(x_{k}, z\right)=A_{k}+\epsilon\left(\sum_{n=1}^{\infty} \hat{B}_{n} \psi_{n}(z)\right)+O\left(\epsilon^{2}\right), \\
\hat{p}\left(x_{k+1}, z\right)=A_{k}+\epsilon\left(\tilde{\sigma} A_{k+1}^{-}{ }^{\prime}+\sum_{n=1}^{\infty} \hat{C}_{n} \psi_{n}(z)\right)+O\left(\epsilon^{2}\right),
\end{gathered}
$$

with $\hat{B}_{n}$ and $\hat{C}_{n}, n=1,2, \ldots$, as given in (3.88) and (3.89). (Note in the above that $A_{k}+\epsilon \tilde{\sigma} A_{k+1}^{-}{ }^{\prime}=A_{k}+\epsilon \tilde{\sigma} A_{k}^{+{ }^{\prime}}+O\left(\epsilon^{2}\right)=A_{k+1}+O\left(\epsilon^{2}\right)$.)

The asymptotic solution to [SSP] as $\epsilon \rightarrow 0$, uniformly for $(x, z) \in \bar{M}^{\prime}$, is now complete. We now turn our attention to the eigenvalue problem [EVP].

4. Asymptotic solution to the eigenvalue problem [EVP] as $\epsilon \rightarrow 0$. In this section we develop the asymptotic solution to the eigenvalue problem [EVP] as $\epsilon \rightarrow 0$. We first employ the theory developed by Ramm [16] to establish that the set of eigenvalues to [EVP], (2.42), with $\epsilon>0$, splits into two disjoint subsets as $\epsilon \rightarrow 0^{+}$, denoted by $S_{-}=\left\{\lambda_{0}^{-}(\epsilon), \lambda_{1}^{-}(\epsilon), \lambda_{2}^{-}(\epsilon), \ldots\right\}$ and $S_{+}=\left\{\lambda_{1}^{+}(\epsilon), \lambda_{2}^{+}(\epsilon), \lambda_{3}^{+}(\epsilon), \ldots\right\}$, with $0=\lambda_{0}^{-}(\epsilon)<\lambda_{1}^{-}(\epsilon)<\cdots$ and $0<\lambda_{1}^{+}(\epsilon)<\lambda_{2}^{+}(\epsilon)<\cdots$. In particular,

$$
\lambda_{n}^{-}(\epsilon)=O\left(n^{2}\right), \quad \lambda_{n}^{+}(\epsilon)=O\left(n^{2} \epsilon^{-2}\right)
$$

as $\epsilon \rightarrow 0^{+}$, uniformly for $n=1,2, \ldots$ We will focus attention on the eigenvalues and corresponding eigenfunctions in the set $S_{-}$, so that in [EVP] we have $\lambda(\epsilon)=O(1)$ as $\epsilon \rightarrow 0^{+}$, via (4.1). Thus we expand $\phi: \bar{M}^{\prime} \mapsto \mathbb{R}$ in the form

$$
\phi(x, z ; \epsilon)=\tilde{\phi}(x, z)+\epsilon^{2} \hat{\phi}(x, z)+o\left(\epsilon^{2}\right) \quad \text { as } \epsilon \rightarrow 0^{+},
$$


uniformly for $(x, z) \in \bar{M}^{\prime}$, while we expand

$$
\lambda(\epsilon)=\tilde{\lambda}+\epsilon^{2} \hat{\lambda}+o\left(\epsilon^{2}\right) \quad \text { as } \epsilon \rightarrow 0^{+} .
$$

On substitution from (4.2) and (4.3) into [EVP], we obtain the leading order problem

$$
\begin{gathered}
\left(D_{z}(x, z) \tilde{\phi}_{z}\right)_{z}=0, \quad(x, z) \in M^{\prime}, \\
\tilde{\phi}_{x}(-1, z)=0, \quad z \in\left[z_{-}(-1), z_{+}(-1)\right], \\
\tilde{\phi}_{x}(1, z)=0, \quad z \in\left[z_{-}(1), z_{+}(1)\right], \\
\tilde{\phi}_{z}\left(x, z_{+}(x)\right)=0, \quad x \in(-1,1), \\
\tilde{\phi}_{z}\left(x, z_{-}(x)\right)=0, \quad x \in(-1,1) .
\end{gathered}
$$

A direct integration of (4.4) gives

$$
\tilde{\phi}_{z}(x, z)=\frac{\tilde{B}(x)}{D_{z}(x, z)}, \quad(x, z) \in \bar{M}^{\prime},
$$

while (4.7) and (4.8) require $\tilde{B}(x)=0$ for all $x \in[-1,1]$. Hence, from (4.9),

$$
\tilde{\phi}(x, z)=\tilde{A}(x), \quad(x, z) \in \bar{M}^{\prime},
$$

with $\tilde{A}:[-1,1] \mapsto \mathbb{R}$ such that $\tilde{A} \in C^{1}([-1,1]) \cap C^{2}((-1,1))$. Conditions (4.5) and (4.6) then require $\tilde{A}^{\prime}(-1)=\tilde{A}^{\prime}(1)=0$. At $O\left(\epsilon^{2}\right)$ we obtain the problem

$$
\begin{gathered}
\left(D_{z}(x, z) \hat{\phi}_{z}\right)_{z}=-\tilde{\lambda} \tilde{A}(x)-\left(D_{x}(x, z) \tilde{A}^{\prime}(x)\right)_{x}, \quad(x, z) \in M^{\prime}, \\
\hat{\phi}_{x}(-1, z)=0, \quad z \in\left[z_{-}(-1), z_{+}(-1)\right], \\
\hat{\phi}_{x}(1, z)=0, \quad z \in\left[z_{-}(1), z_{+}(1)\right], \\
D_{z}\left(x, z_{+}(x)\right) \hat{\phi}_{z}\left(x, z_{+}(x)\right)=z_{+}^{\prime}(x) D_{x}\left(x, z_{+}(x)\right) \tilde{A}^{\prime}(x), \quad x \in(-1,1), \\
D_{z}\left(x, z_{-}(x)\right) \hat{\phi}_{z}\left(x, z_{-}(x)\right)=z_{-}^{\prime}(x) D_{x}\left(x, z_{-}(x)\right) \tilde{A}^{\prime}(x), \quad x \in(-1,1) .
\end{gathered}
$$

The solvability requirement on the inhomogeneous boundary value problem (4.11)(4.15) will provide the ordinary differential equation which must be satisfied by $\tilde{A}(x)$, $x \in(-1,1)$. On integrating (4.11) with respect to $z$ (with $x \in(-1,1)$ fixed) between $z=z_{-}(x)$ and $z=z_{+}(x)$, we obtain

$$
\begin{gathered}
D_{z}\left(x, z_{+}(x)\right) \hat{\phi}_{z}\left(x, z_{+}(x)\right)-D_{z}\left(x, z_{-}(x)\right) \hat{\phi}_{z}\left(x, z_{-}(x)\right)=-\tilde{\lambda} \tilde{A}(x)\left(z_{+}(x)-z_{-}(x)\right) \\
-\left(\bar{D}_{x}(x) \tilde{A}^{\prime}(x)\right)^{\prime}+\left[z_{+}^{\prime}(x) D_{x}\left(x, z_{+}(x)\right)-z_{-}^{\prime}(x) D_{x}\left(x, z_{-}(x)\right)\right] \tilde{A}^{\prime}(x)
\end{gathered}
$$

for all $x \in(-1,1)$. We next substitute into the left-hand side of (4.16) from (4.14) and (4.15), which, after cancellation, results in the ordinary differential equation

$$
\left(\bar{D}_{x}(x) \tilde{A}^{\prime}(x)\right)^{\prime}+\tilde{\lambda}\left(z_{+}(x)-z_{-}(x)\right) \tilde{A}(x)=0, \quad x \in(-1,1) .
$$

Thus $\tilde{A}:[-1,1] \mapsto \mathbb{R}$ and $\tilde{\lambda} \in \mathbb{R}$ satisfy the regular Sturm-Liouville eigenvalue problem (which we denote hereafter by [SLP]),

$$
\begin{gathered}
\left(\bar{D}_{x}(x) \tilde{A}^{\prime}(x)\right)^{\prime}+\tilde{\lambda} h(x) \tilde{A}(x)=0, \quad x \in(-1,1), \\
\tilde{A}^{\prime}(-1)=\tilde{A}^{\prime}(1)=0,
\end{gathered}
$$


with $\bar{D}_{x}(x)$ as defined in (3.18) and $h(x)=z_{+}(x)-z_{-}(x), x \in[-1,1]$. Now, the classical Sturm-Liouville theory (see, for example, [6, Chapters 7, 8]) determines that the set of eigenvalues of [SLP] is given by $\tilde{\lambda}=\tilde{\lambda}_{r}, r=0,1,2, \ldots$, with

$$
0=\tilde{\lambda}_{0}<\tilde{\lambda}_{1}<\tilde{\lambda}_{2}<\cdots \quad \text { and } \quad \tilde{\lambda}_{r}=O\left(r^{2}\right) \quad \text { as } r \rightarrow \infty .
$$

We remark also that [SLP] is identical in structure to the eigenvalue problem [SL] considered in section 3 . Corresponding to each eigenvalue $\tilde{\lambda}_{r}(r=0,1,2, \ldots)$, there is a unique normalized eigenfunction $\tilde{A}_{r}:[-1,1] \mapsto \mathbb{R}$ such that

$$
\int_{-1}^{1} h(x) \tilde{A}_{i}(x) \tilde{A}_{j}(x) \mathrm{d} x=\delta_{i j} \quad \text { for } i, j=0,1,2, \ldots
$$

We note that $\tilde{A}_{0}(x)=\left\{\int_{-1}^{1} h(s) \mathrm{d} s\right\}^{-1 / 2}=\left(\operatorname{meas}\left(\bar{M}^{\prime}\right)\right)^{-1 / 2}$ for all $x \in[-1,1]$. Thus, we have established for [EVP], via (4.2), (4.3), (4.10), that $\lambda_{r}^{-}(\epsilon)=\tilde{\lambda}_{r}+O\left(\epsilon^{2}\right)$ as $\epsilon \rightarrow 0$, uniformly for $r=1,2, \ldots$, with corresponding normalized eigenfunction $\phi_{r}^{-}(x, z ; \epsilon)=\tilde{A}_{r}(x)+O\left(\epsilon^{2}\right)$ as $\epsilon \rightarrow 0$, uniformly for $(x, z) \in \bar{M}^{\prime}$.

We can now use the above theory to obtain the following expression for $\tilde{p}$ : $\bar{M}^{\prime} \times[0, \infty) \mapsto \mathbb{R}$, via $(2.46)$ and $(2.47)$ :

$$
\tilde{p}(x, z, t)=\sum_{r=1}^{\infty} c_{r} \mathrm{e}^{-\tilde{\lambda}_{r} t} \tilde{A}_{r}(x)+O\left(\epsilon^{2} \mathrm{e}^{-\tilde{\lambda}_{1} t}, \mathrm{e}^{-t / \epsilon^{2}}\right) \quad \text { as } \epsilon \rightarrow 0,
$$

uniformly for $(x, z, t) \in \bar{M}^{\prime} \times[\delta, \infty)$, for any $\delta>0$. Here $c_{r}, r=1,2, \ldots$, are given by

$$
c_{r}=\iint_{\bar{M}^{\prime}} \tilde{p}_{0}(u, v) \tilde{A}_{r}(u) \mathrm{d} u \mathrm{~d} v, \quad r=1,2, \ldots
$$

We observe from (4.20) that

$$
\tilde{p}(x, z, t) \sim\left(c_{1} \tilde{A}_{1}(x)+O\left(\epsilon^{2}\right)\right) \mathrm{e}^{-\tilde{\lambda}_{1} t}
$$

as $t \rightarrow \infty$, uniformly for $(x, z) \in \bar{M}^{\prime}$. Thus, the solution to [IBVP] approaches the solution to [SSP] as $t \rightarrow \infty$ through terms exponentially small in $t$ as $t \rightarrow \infty$. The timescale for relaxation to the steady state is then $t_{s} \sim\left(\tilde{\lambda}_{1}\right)^{-1}$ in dimensionless variables, giving the dimensional relaxation timescale as $t_{s}^{d} \sim c_{t} l^{2} /\left(D_{0} \tilde{\lambda}_{1}\right)$, via (2.12).

5. The case of disparate permeabilities. In the previous sections, the theory has been developed for the situation when the permeability scale is comparable in both the $x$-direction and the $z$-direction. In some applications, this is not always the case, when the permeability in the $z$-direction is much weaker than that in the $x$-direction. In this case (2.1) should be replaced by

$$
D_{0}^{x} D_{x}\left(\frac{x}{l}, \frac{z}{h}\right) \geq D_{m}>0, \quad D_{0}^{z} D_{z}\left(\frac{x}{l}, \frac{z}{h}\right) \geq D_{m}>0,
$$

for $(x, z) \in \bar{M}$, with $D_{0}^{x}>0$ being the permeability scale in the $x$-direction and $D_{0}^{z}>0$ being the permeability scale in the $z$-direction, and now

$$
0<\delta=\frac{D_{0}^{z}}{D_{0}^{x}} \ll 1 .
$$

For such reservoirs $\delta$ is typically of $O\left(10^{-1}\right)$. We now follow the same nondimensionalization as before, via (2.12), with $D_{0}^{x}$ replacing $D_{0}$. The resulting full dimensionless 
problem is identical to [IBVP], except with $\epsilon$ replaced by $\tilde{\epsilon}$, where $\tilde{\epsilon}=\epsilon \delta^{-1 / 2}$. Thus all of the previous theory carries over to this situation, on simply replacing $\epsilon$ by $\tilde{\epsilon}$. In particular, the asymptotic theory as $\epsilon \rightarrow 0$ is now replaced by $\tilde{\epsilon} \rightarrow 0$, and so requires

$$
0<\tilde{\epsilon} \ll 1
$$

which, with (5.1), is equivalent to $0<\epsilon \ll \delta^{1 / 2} \ll 1$, and with typically $\epsilon^{2} \sim O\left(10^{-4}\right)$ and $\delta \sim O\left(10^{-1}\right)$, then this ordering is satisfied. It is worth noting here that for porous layers in which $\epsilon=O(1)$ and $\delta \gg 1$, then $\tilde{\epsilon}=\epsilon \delta^{-1 / 2} \ll 1$, so (5.2) is again satisfied, and the asymptotic theory developed before is again applicable.

6. The pseudosteady state. In this section we consider the situation when the specified flux constants $\alpha_{i}, i=1, \ldots, N$, do not satisfy the condition (2.31); that is, when $\sum_{i=1}^{N} \alpha_{i}=\alpha_{T} \neq 0$. In this case we introduce an associated pseudosteady state problem to [IBVP]. This corresponds to the steady state problem [SSP] ((2.23)(2.30)), except that now (2.23) is modified to

$$
\hat{u}_{x}+\hat{w}_{z}=\sum_{i=1}^{N} s_{i}(z) \delta\left(x-x_{i}\right)-\tilde{\alpha}_{T}, \quad(x, z) \in M^{\prime},
$$

with the constant $\tilde{\alpha}_{T}$ given by $\tilde{\alpha}_{T}=\alpha_{T} /$ meas $\left(\bar{M}^{\prime}\right)$. The result corresponding to Theorem 2.2 is now, with the pseudosteady state problem referred to as [PSSP], the following.

THEOREM 6.1. For each $\epsilon>0$, [PSSP] has a unique (up to addition of a constant in $\hat{p}$ ) solution $\hat{u}, \hat{w}, \hat{p}: \bar{M}^{\prime} \mapsto \mathbb{R}$.

Again, we fix the indeterminate constant in [PSSP] to be that pseudosteady state which satisfies the condition (2.40). The result corresponding to Theorem 2.3 is now as follows.

TheOREM 6.2. For each $\epsilon>0$, [IBVP] has a unique solution $u, w, \bar{p}: \bar{M}^{\prime} \times$ $[0, \infty) \mapsto \mathbb{R}$ given by

$$
\begin{aligned}
& \bar{p}(x, z, t)=\tilde{\alpha}_{T} t+\hat{p}(x, z)+\tilde{p}(x, z, t), \\
& u(x, z, t)=\hat{u}(x, z)-D_{x}(x, z) \tilde{p}_{x}(x, z, t), \\
& w(x, z, t)=\hat{w}(x, z)-\epsilon^{-2} D_{z}(x, z) \tilde{p}_{z}(x, z, t),
\end{aligned}
$$

for all $(x, z) \in \bar{M}^{\prime}$ and $t \in[0, \infty)$. Here $\tilde{p}: \bar{M}^{\prime} \times[0, \infty) \mapsto \mathbb{R}$ is given by (2.46), (2.47), and $\hat{u}, \hat{w}, \hat{p}: \bar{M}^{\prime} \mapsto \mathbb{R}$ is that solution to [PSSP] which satisfies (2.40). Moreover,

$$
\begin{aligned}
\bar{p}(x, z, t) & =\tilde{\alpha}_{T} t+\hat{p}(x, z)+O\left(\mathrm{e}^{-\lambda_{1}(\epsilon) t}\right), \\
u(x, z, t) & =\hat{u}(x, z)+O\left(\mathrm{e}^{-\lambda_{1}(\epsilon) t}\right), \\
w(x, z, t) & =\hat{w}(x, z)+O\left(\mathrm{e}^{-\lambda_{1}(\epsilon) t}\right),
\end{aligned}
$$

as $t \rightarrow \infty$, uniformly for $(x, z) \in \bar{M}^{\prime}$.

We remark that since $\hat{p} \in C\left(\bar{M}^{\prime}\right) \cap P C^{1}\left(\bar{M}^{\prime}\right)$ and the initial data for [IBVP] $\bar{p}_{0} \in C\left(\bar{M}^{\prime}\right) \cap P C^{1}\left(\bar{M}^{\prime}\right)$, then Theorem 6.2 implies global asymptotic stability (up to the addition of a constant to $\hat{p}$ ) for the pseudosteady state to [IBVP] with respect to perturbations in $C\left(\bar{M}^{\prime}\right) \cap P C^{1}\left(\bar{M}^{\prime}\right)$.

We now explore the structure of the solution to [PSSP] as $\epsilon \rightarrow 0$. This involves only minor adjustments to the structure developed in section 3 for the solution to 
[SSP]. Again the outer region asymptotic expansions are given by

$$
\begin{aligned}
\hat{u}(x, z ; \epsilon)= & \frac{-D_{x}(x, z)}{\bar{D}_{x}(x)}\left\{S(x)+\tilde{\alpha}_{T} H(x)\right\}+O\left(\epsilon^{2}\right), \\
\hat{w}(x, z ; \epsilon)= & S(x) \int_{z_{-}(x)}^{z}\left\{\frac{D_{x}(x, \lambda)}{\bar{D}_{x}(x)}\right\}_{x} \mathrm{~d} \lambda \\
& \quad-\frac{z_{-}^{\prime}(x) D_{x}\left(x, z_{-}(x)\right) S(x)}{\bar{D}_{x}(x)}-\tilde{\alpha}_{T}\left(z-z_{-}(x)\right)+O\left(\epsilon^{2}\right), \\
\hat{p}(x, z ; \epsilon)= & A(x)+O\left(\epsilon^{2}\right),
\end{aligned}
$$

as $\epsilon \rightarrow 0$, uniformly for $(x, z) \in \bar{N}_{\epsilon}^{\prime}$. Here, as before, $S:[-1,1] \mapsto \mathbb{R}$ is as given by (3.20), but now $A:[-1,1] \mapsto \mathbb{R}$ is the unique solution to the linear inhomogeneous boundary value problem,

$$
\begin{aligned}
{\left[\bar{D}_{x}(x) A^{\prime}(x)\right]^{\prime}=} & -\sum_{i=1}^{N} \alpha_{i} \delta\left(x-x_{i}\right)+\tilde{\alpha}_{T}\left(z_{+}(x)-z_{-}(x)\right), \quad x \in(-1,1), \\
A^{\prime}(-1)=A^{\prime}(1)=0, & \int_{-1}^{1}\left(z_{+}(x)-z_{-}(x)\right) A(x) \mathrm{d} x=I_{0},
\end{aligned}
$$

with $H:[-1,1] \mapsto \mathbb{R}$ defined by

$$
H(x)=\int_{-1}^{x}\left(z_{+}(\lambda)-z_{-}(\lambda)\right) \mathrm{d} \lambda, \quad x \in[-1,1] .
$$

The solution to (6.2)-(6.4) is readily obtained as

$$
A(x)=\int_{-1}^{x} \frac{\left[S(\lambda)+\tilde{\alpha}_{T} H(\lambda)\right]}{\bar{D}_{x}(\lambda)} \mathrm{d} \lambda+A_{0}, \quad x \in[-1,1],
$$

with the constant $A_{0}$ given by

$$
A_{0}=\frac{I_{0}}{\operatorname{meas}\left(\bar{M}^{\prime}\right)}-\frac{1}{\operatorname{meas}\left(\bar{M}^{\prime}\right)} \int_{-1}^{1} \frac{\left[S(\lambda)+\tilde{\alpha}_{T} H(\lambda)\right]}{\bar{D}_{x}(\lambda)} \operatorname{meas}\left(\bar{M}^{\prime}(\lambda)\right) \mathrm{d} \lambda .
$$

Thus we now have

$$
\begin{gathered}
A_{i}=A\left(x_{i}\right)=\int_{-1}^{x_{i}} \frac{\left[S(\lambda)+\tilde{\alpha}_{T} H(\lambda)\right]}{\bar{D}_{x}(\lambda)} \mathrm{d} \lambda+A_{0}, \\
{A_{i}^{+}}^{\prime}=A^{\prime}\left(x_{i}^{+}\right)=-\frac{\sum_{j=0}^{i} \alpha_{j}}{\bar{D}_{x}\left(x_{i}\right)}+\frac{\tilde{\alpha}_{T} H\left(x_{i}\right)}{\bar{D}_{x}\left(x_{i}\right)}, \\
A_{i}^{{ }^{\prime}}=A^{\prime}\left(x_{i}^{-}\right)=-\frac{\sum_{j=0}^{i-1} \alpha_{j}}{\bar{D}_{x}\left(x_{i}\right)}+\frac{\tilde{\alpha}_{T} H\left(x_{i}\right)}{\bar{D}_{x}\left(x_{i}\right)},
\end{gathered}
$$

each for $i=1, \ldots, N$. The details of the inner regions are precisely as before in section 3 , but now $A_{i}$ and $A_{i}^{ \pm^{\prime}}$ are given by (6.8)-(6.10). The modifications are now complete. 
7. An example. We finally apply the theory developed in the previous sections to a simple situation. We consider a rectangular porous layer, with $z_{ \pm}(x)= \pm 1 / 2$, $x \in[-1,1]$, with the permeability of the layer in the $x$-direction independent of $x$, so that $D_{x}(x, z)=D_{x}(z),(x, z) \in \bar{M}^{\prime}$. Thus,

$$
\bar{D}_{x}(x)=\int_{-1 / 2}^{1 / 2} D_{x}(z) \mathrm{d} z=\bar{D}_{x}(>0), \quad x \in[-1,1]
$$

with $\bar{D}_{x}$ a constant. We include a single source/sink at $x=x_{1}=x_{s} \in(-1,1)$, with normalized flux constant $\alpha=\alpha_{1}=\alpha_{s}= \pm 1$, with -1 for extraction and +1 for injection. We take the initial pressure field to be uniform, so that

$$
\bar{p}_{0}(x, z)=\bar{p}_{0}, \quad(x, z) \in \bar{M}^{\prime},
$$

with $\bar{p}_{0}$ a constant. The pressure field in the porous layer is then given by

$$
\bar{p}(x, z, t ; \epsilon)=\frac{1}{2} \alpha_{s} t+A(x)+c_{1} \tilde{A}_{1}(x) \mathrm{e}^{-\tilde{\lambda}_{1} t}+O\left(\epsilon, \mathrm{e}^{-\tilde{\lambda}_{2} t}, \epsilon^{2} \mathrm{e}^{-\tilde{\lambda}_{1} t}, \mathrm{e}^{-t / \epsilon^{2}}\right),
$$

as $\epsilon \rightarrow 0$, uniformly for $(x, z, t) \in \bar{M}^{\prime} \times[\delta, \infty)$ (for any $\delta>0$ ), via Theorem 6.2, together with (6.1), (3.34), (3.41), and (4.20)-(4.22). From (4.17)-(4.19),

$$
\tilde{\lambda}_{1}=\frac{1}{4} \bar{D}_{x} \pi^{2}, \quad \tilde{\lambda}_{2}=\bar{D}_{x} \pi^{2}, \quad \tilde{A}_{1}(x)=\cos \frac{1}{2} \pi(x+1), \quad x \in[-1,1],
$$

with, via (4.21), (7.1), and (6.1),

$$
c_{1}=-\int_{-1}^{1} A(\lambda) \cos \frac{1}{2} \pi(\lambda+1) \mathrm{d} \lambda .
$$

It also follows from (6.2)-(6.7) that

$$
A(x)= \begin{cases}\frac{\alpha_{s}}{4 D_{x}}(x+1)^{2}+A_{0}, & x \in\left[-1, x_{s}\right), \\ \frac{\alpha_{s}}{4 D_{x}}(x+1)^{2}-\frac{\alpha_{s}}{D_{x}}\left(x-x_{s}\right)+A_{0}, & x \in\left[x_{s}, 1\right],\end{cases}
$$

with $A_{0}=\bar{p}_{0}-\frac{\alpha_{s}}{12 D_{x}}\left(1+6 x_{s}-3 x_{s}^{2}\right)$. The pressure at the line source/sink is thus

$$
\bar{p}\left(x_{s}, z, t ; \epsilon\right)=\frac{1}{2} \alpha_{s} t+A\left(x_{s}\right)+c_{1} \tilde{A}_{1}\left(x_{s}\right) \mathrm{e}^{-\tilde{\lambda}_{1} t}+O\left(\epsilon, \mathrm{e}^{-\tilde{\lambda}_{2} t}, \epsilon^{2} \mathrm{e}^{-\tilde{\lambda}_{1} t}, \mathrm{e}^{-t / \epsilon^{2}}\right),
$$

from which it follows, via (7.3)-(7.5), that

$$
\begin{gathered}
\bar{p}\left(x_{s}, z, t ; \epsilon\right)=\frac{1}{2} \alpha_{s} t+\bar{p}_{0}+\frac{\alpha_{s}}{6 \bar{D}_{x}}\left(1+3 x_{s}^{2}\right)+c_{1} \mathrm{e}^{-\frac{\bar{D}_{x} \pi^{2}}{4} t} \cos \frac{1}{2} \pi\left(x_{s}+1\right) \\
+O\left(\epsilon, \mathrm{e}^{-\bar{D}_{x} \pi^{2} t}, \epsilon^{2} \mathrm{e}^{-\frac{\bar{D}_{x} \pi^{2}}{4} t}, \mathrm{e}^{-t / \epsilon^{2}}\right)
\end{gathered}
$$

as $\epsilon \rightarrow 0$, uniformly for $(z, t) \in[-1 / 2,1 / 2] \times[\delta, \infty)$. To obtain the correction at $O(\epsilon)$ to (7.2), we note that a composite expansion must first be obtained using the inner and outer expansions for $\bar{p}$, but this is not necessary at leading order. As $t \rightarrow \infty$, (7.6) gives

$$
\bar{p}\left(x_{s}, z, t ; \epsilon\right) \sim \bar{p}_{0}+\frac{1}{2} \alpha_{s}\left[t+\frac{1}{3 \bar{D}_{x}}\left(1+3 x_{s}^{2}\right)\right]
$$


Finally, denoting the dimensionless atmospheric pressure by $\bar{p}_{a}$, then with the initial layer pressure $\bar{p}_{0} \gg \bar{p}_{a}$, we may use (7.7) to obtain the time span during which the single well is self-producing at the specified extraction rate. With an extraction well, $\alpha_{s}=-1$ in (7.7), and the time limit of self-production is $t=t_{c}$, where

$$
\bar{p}\left(x_{s}, z, t_{c} ; \epsilon\right)=\bar{p}_{a} .
$$

On using (7.7) in (7.8), we obtain

$$
t_{c}=2\left(\bar{p}_{0}-\bar{p}_{a}\right)-\frac{1}{3 \bar{D}_{x}}\left(1+3 x_{s}^{2}\right) .
$$

It follows from (7.9) that $t_{c}$ is optimized by locating the extraction well at $x=x_{s}=0$, that is, at the center of the porous layer as should be expected due to the symmetry in this simple example. The point is that, in less symmetrical examples, optimization can be achieved with little more effort through the corresponding version of (7.9), which is still readily available. In dimensional terms, (7.9) becomes, via (2.12),

$$
t_{c}^{d}=\frac{a c_{t}}{Q}\left(p_{0}^{d}-p_{a}\right)-\frac{h c_{t}}{3 \bar{D}_{x}^{d}}\left(l^{2}+3 x_{s}^{d^{2}}\right),
$$

with $t_{c}^{d}$ the dimensional self-extraction time, $a=2 h l$ the cross-sectional area of the porous layer, $Q$ the volumetric extraction rate per unit width, $p_{0}^{d}$ the dimensional initial layer pressure, $p_{a}$ the dimensional atmospheric pressure, $\bar{D}_{x}^{d}$ the dimensional depth integrated permeability in the $x$-direction, and $x_{s}^{d}$ the dimensional location of the extraction well.

8. Conclusions. In this paper we have considered the unsteady flow of a weakly compressible fluid in a horizontal layer of an inhomogeneous and anisotropic porous medium with variable upper and lower boundaries, in the presence of line sources and sinks. We have derived a strongly parabolic linear initial boundary value problem for the dynamic fluid pressure and shown that this problem has a unique solution. We have then constructed the solution to this problem when the layer aspect ratio $0<\epsilon \ll 1$, via the method of matched asymptotic expansions. First, we have derived a matched asymptotic solution to the steady state problem, under the constraint that the sum of the total volume fluxes at the wells is zero. (This constraint is removed in section 6 , leading to a pseudosteady state problem whose solution is almost identical in structure.) In the outer region this has been constructed directly, with the solution given by (3.30)-(3.32). In the inner region the solution is given by (3.63)-(3.66), together with (3.34) and (3.41). This solution is written in terms of the eigenvalues and eigenvectors of a regular Sturm-Liouville eigenvalue problem [SL], which can be solved analytically in the case that the permeability at each line source/sink is constant in the vertical direction, but whose numerical solution is straightforward in the more general case. The pressure at any line source or sink is then given by (3.67).

By subtracting the solution of the steady state problem from the solution of the initial value problem, we have then constructed a strongly parabolic homogeneous problem with no discontinuities across the line sources and sinks, whose solution can be written in terms of the eigenvalues and eigenfunctions of a regular self-adjoint eigenvalue problem. Asymptotic solution of this reduces to solution of a regular Sturm-Liouville eigenvalue problem identical in structure to [SL]. It has further been shown, via (4.20)-(4.22), that the solution of the initial value problem approaches the solution of the steady state problem through terms exponentially small with respect 
to time $t$ as $t \rightarrow \infty$. Generalizations to cases where a line source or sink is near a boundary wall, where line sources and sinks are not well spaced, and to the case of disparate permeabilities have also been considered, in sections 3.1, 3.2, and 5, respectively. An example demonstrating an application of the theory to a simple situation is provided in section 7 .

We finally remark that since the initial boundary value problem is solved for a general $C^{1}$ initial condition, the effect of time dependent transient effects due to temporal changes in the well discharge rates can easily be accounted for.

\section{REFERENCES}

[1] A.-J. A. Al-Khalifah, K. Aziz, And R. N. Horne, A new approach to multiphase well test analysis, in Society of Petroleum Engineers 16743, SPE, Houston, TX, 1987.

[2] K. Aziz And A. Settari, Petroleum Reservoir Simulation, Applied Science Publishers, London, 1979.

[3] D. K. Babu And A. S. Odeh, Productivity of a horizontal well, in Society of Petroleum Engineers 18334, SPE, Houston, TX, 1988.

[4] G. S. Busswell, R. Banerjee, R. K. M. Thambynayagam, and J. B. Spath, Generalized analytical solution for reservoir problems with multiple wells and boundary conditions, in Society of Petroleum Engineers 99288, SPE, Houston, TX, 2006.

[5] H. Cinco-Ley, F. Samaniego-V, And A. N. Dominguez, Transient pressure behavior for a well with a finite-conductivity vertical fracture, Soc. Petroleum Engineers J., 18 (1978), pp. $253-264$.

[6] E. A. Coddington and N. Levinson, Theory of Ordinary Differential Equations, McGrawHill, New York, Toronto, London, 1955.

[7] A. Friedman, Partial Differential Equations of Parabolic Type, Prentice-Hall, Englewood Cliffs, NJ, 1964.

[8] J. P. Gilchrist, G. S. Busswell, R. Banerjee, J. B. Spath, and R. K. M. Thambynayagam, Semi-analytical solution for multiple layer problems with multiple vertical, horizontal, deviated and fractured wells, in Proceedings of the International Petroleum Technology Conference, Dubai, U.A.E., 2007, SPE, Houston, TX, paper 11718.

[9] E. Gomes And Z. A. RezA, A new semi-analytic pressure transient model for layered gas reservoir under various reservoir and well conditions, in Society of Petroleum Engineers 39526, SPE, Houston, TX, 1998.

[10] A. Gringarten and H. J. Ramey, JR., The use of source and Green's functions in solving unsteady-flow problems in reservoirs, Soc. Petroleum Engineers J., 13 (1973), pp. 285-296.

[11] D. J. Needham and S. Langdon, The Unsteady Flow of a Weakly Compressible Fluid in a Thin Porous Layer. II: Three-dimensional Theory, Mathematics Department Preprint Series MPS_2009_01, University of Reading, UK.

[12] M. Oguztoreli and D. W. Wong, Vertex: A new modeling method to direct field development, in Society of Petroleum Engineers 39806, SPE, Houston, TX, 1998.

[13] E. Ozkhan and R. Raghavan, Well test analysis problems: Part 1-Analytical considerations, in Society of Petroleum Engineers 18615, SPE, Houston, TX, 1991.

[14] R. Pecher, S. D. Harris, R. J. Knipe, L. Elliot, and D. B. Ingham, New formulation of the Green element method to maintain its second order accuracy in 2D/3D, Engineering Analysis with Boundary Elements, 25 (2001), pp. 211-219.

[15] R. Raghavan, Well test analysis: Wells producing by solution gas drive, in Society of Petroleum Engineers 5588, SPE, Houston, TX, 1975.

[16] A. G. RAMm, Limit spectra of the interior Neumann problems when a solid domain shrinks to a plane one, J. Math. Anal. Appl., 108 (1985), pp. 107-112.

[17] J. Smoller, Shock Waves and Reaction-Diffusion Equations, Springer-Verlag, Berlin, 1983.

[18] R. K. M. Thambynayagam, Diffusion: A Compendium of Analytical Solutions, in preparation.

[19] L. G. Thompson, J. L. Manrique, and T. A. Jelmeri, Efficient algorithms for computing the bounded reservoir horizontal well pressure response, in Society of Petroleum Engineers 21827, SPE, Houston, TX, 1991.

[20] M. Van Dyke, Perturbation Methods in Fluid Mechanics, The Parabolic Press, Stanford, CA, 1975. 